\title{
Physics implications of flat directions in free fermionic superstring models. I. Mass spectrum and couplings
}

\author{
G. Cleaver, ${ }^{2, *}$ M. Cvetič ${ }^{2}$ J. R. Espinosa, ${ }^{1}$ L. Everett ${ }^{2, \dagger}$ P. Langacker, ${ }^{2}$ and J. Wang ${ }^{2}$ \\ ${ }^{1}$ CERN, TH Division, CH-1211 Geneva 23, Switzerland \\ ${ }^{2}$ Department of Physics and Astronomy, University of Pennsylvania, Philadelphia Pennsylvania 19104-6396
}

(Received 12 August 1998; published 1 February 1999)

\begin{abstract}
From the "top-down" approach we investigate physics implications of the class of $D$ - and $F$-flat directions formed from non-Abelian singlets which are proved flat to all orders in the nonrenormalizable superpotential, for a prototype quasi-realistic free fermionic string model with the standard model gauge group and three families (CHL5). These flat directions have at least an additional $U(1)^{\prime}$ unbroken at the string scale. For each flat direction, the complete set of effective mass terms and effective trilinear superpotential terms in the observable sector are computed to all orders in the VEV's of the fields in the flat direction. The "string selection rules" disallow a large number of couplings allowed by gauge invariance, resulting in a massless spectrum with a large number of exotics, in most cases excluded by experiment, thus signifying a generic flaw of these models. Nevertheless, the resulting trilinear couplings of the massless spectrum possess a number of interesting features which we analyze for two representative flat directions: for the fermion texture, baryonand lepton-number violating couplings, $R$-parity breaking, non-canonical $\mu$ terms, and the possibility of electroweak and intermediate scale symmetry breaking scenarios for $U(1)^{\prime}$. The gauge coupling predictions are obtained in the electroweak scale case. Fermion masses possess $t-b$ and $\tau$ - $\mu$ universality, with the string scale Yukawa couplings $g$ and $g / \sqrt{2}$, respectively. Fermion textures are present for certain flat directions, but only in the down-quark sector. Baryon- and lepton-number violating couplings can trigger proton decay, $N-\bar{N}$ oscillations, leptoquark interactions and $R$-parity violation, leading to the absence of a stable LSP.
\end{abstract}

[S0556-2821(99)00205-2]

PACS number(s): 12.60.Jv, 11.30.Pb

\section{INTRODUCTION}

At present, there are several challenges to be faced in the investigation of the implications of superstring theory for physics beyond the standard model (SM). A primary obstacle is the degeneracy of string vacua; a large number of string models have been constructed, with as yet no fully realistic model. There is also no satisfactory scenario for supersymmetry breaking in string theory either at the level of the world-sheet dynamics or at the level of the effective theory, and hence no way to break supersymmetry in string models without introducing new parameters. It is hoped that issues will have a resolution with a greater understanding of nonperturbative string dynamics.

Our strategy is to take a more modest view by restricting our consideration to a class of string models of perturbative heterotic string vacua [1-5] which have the ingredients of the minimal supersymmetric standard model (MSSM) and thus the potential to be realistic. Such quasi-realistic models have been constructed in a weakly coupled heterotic superstring theory in a variety of constructions [6-11]. We consider a class of free fermionic models [8-11] which have $N=1$ supersymmetry, the SM gauge group as a part of the gauge structure, and candidate fields for the three generations

\footnotetext{
*Present address: Center for Theoretical Physics, Texas A\&M University, College Station, TX 77843-4242.

${ }^{\dagger}$ Present address: Randall Laboratory of Physics, University of Michigan, Ann Arbor, MI 48109.
}

of quarks and leptons as well as two electroweak Higgs doublets. These models also possess gauge coupling unification at $M_{\text {String }} \sim 5 \times 10^{17} \mathrm{GeV}$ [12] without a gauge group unification; this scale differs by an order of magnitude from the unification scale obtained by extrapolating from the observed low-energy values of the gauge couplings assuming the minimal particle content of the minimal supersymmetric standard model (MSSM). (For a review of the properties of string models, see Ref. [13] and references therein.)

These models share a number of generic features. Their gauge structures contain at the tree level an additional nonAbelian "hidden" sector gauge group as well as a number of Abelian gauge groups, one of them generically anomalous. The SM hypercharge is determined as a linear combination of the non-anomalous $U(1)$ 's of the model [or perhaps of the $U(1)$ 's that arise when the hidden sector gauge group is broken]. In addition to the MSSM fields, the particle content typically includes a number of fields which are nontrivial representations under the SM (observable sector) gauge group or the non-Abelian hidden sector gauge group (or both), as well as a number of non-Abelian singlet fields. Most of the fields of a given model are charged under the $U(1)$ gauge groups, such that in general there is no distinct separation between the observable and hidden sector gauge groups.

In this class of models, the couplings are calculable in string theory; techniques have been developed to calculate the superpotential $[14-16,11,17,18]$ in principle to all orders in nonrenormalizable terms. One generic feature of the superpotentials is that additional world-sheet selection rules 
forbid terms otherwise allowed by gauge invariance. However, the determination of the Kähler potential is more involved in part because the Kähler potential is not protected by supersymmetric non-renormalization theorems and thus receives corrections at all orders in the string loop expansion. Therefore, in the analysis that follows we assume a minimal Kähler potential, for the sake of simplicity.

The analysis of this class of quasi-realistic models proceeds in several stages. The first step is to address the presence of the anomalous $U(1)$ in the model. The underlying superstring theory is anomaly-free, and hence there is a standard mechanism in the four-dimensional effective theory in which the axion-dilaton supermultiplet shifts under $U(1)_{A}$ in such a way that all triangle anomalies are cancelled. The anomaly cancellation mechanism generates a nonzero FayetIliopoulos (FI) contribution to the $D$ term of the anomalous $U(1)$ at higher genus in string theory [19-21]. The FI term would break supersymmetry in the original string vacuum, but certain scalar fields are triggered to acquire large vacuum expectation values (VEV's) along $D$ - and $F$-flat directions $[19,22]$. The new "restabilized" vacuum is supersymmetric, with a gauge structure of reduced rank [in particular, the anomalous $U(1)$ is broken] and a reduced number of massless fields, as the fields which couple to the fields in the flat direction can acquire string-scale masses and decouple from the theory. Therefore, an analysis of the $D$ - and $F$-flat directions is the necessary first step in the investigation of the phenomenology of the string model. [Issues of anomalous $U(1)$ in string models and string (motivated) models are discussed in [23,24].]

In a previous paper [17], we developed techniques to classify the flat directions of a general perturbative heterotic superstring model with an anomalous $U(1)$. For the sake of simplicity, we chose to consider flat directions formed of non-Abelian singlets only, and selected the singlet fields with zero hypercharge to preserve the SM gauge group. Our method involves classifying the fields according to their anomalous charge to see if flat directions that can cancel the FI term can be formed. If such flat directions can be formed, we construct the superbasis of all one-dimensional (i.e., that which depend on one free VEV before imposing the anomalous $D$ term constraint) $D$-flat directions under the nonanomalous $U(1)$ 's. The elements of the superbasis with the appropriate sign of the anomalous charge to cancel the FI term are the building blocks of the $D_{A}$-flat directions of the model.

For a subset of these $D$-flat directions, the requirements of gauge invariance as well as a string calculation of the superpotential to a given order suffice to prove $F$-flatness to all orders in the nonrenormalizable superpotential. Our method provides a systematic and complete classification of the subset of the $D$-flat directions which can be proved to be $F$-flat to all orders. Each of these flat directions corresponds to a new restabilized string vacuum of a given model. We applied our method to a prototype string model, model 5 of [11] (CHL5), in [17], and more recently to a number of free fermionic string models in [18].

The next stage of the analysis of this class of string models is to investigate the implications of each flat direction of a given model. In general, a number of $U(1)$ 's are broken in each flat direction, though we find that usually at least one $U(1)$ in addition to $U(1)_{Y}$ remains unbroken. The couplings of the fields in the flat direction to the other fields in the model lead to the generation of effective mass terms, such that some of the fields acquire superheavy masses and decouple from the theory. Effective trilinear couplings are also induced from higher-dimensional terms for the remaining light states, with implications for the phenomenology of the model. Effective nonrenormalizable terms are also generated. ${ }^{1}$ We have developed techniques to determine the effective bilinear and trilinear couplings to all orders in the fields with nonzero VEV's along a particular flat direction. Once these terms are determined, they are exact to all orders in the string genus expansion [26]. The details of the effective superpotential strongly depend on the flat direction under consideration.

In this paper, we analyze a class of the flat directions obtained in [17] for the prototype string model [model 5 in [11] (CHL5)], and investigate the implications of the mass spectrum and effective trilinear couplings. We choose to consider flat directions with the maximal number of fields, which also break the maximal number of $U(1)$ 's; in these directions, one $U(1)^{\prime}$ is unbroken in addition to $U(1)_{Y}$. We generalize our techniques developed to prove $F$-flatness to determine the effective renormalizable superpotential:

Massless spectrum. For each flat direction, we determine the complete massless spectrum (at the string scale). We find that the flat directions considered share the undesirable feature that along with the MSSM content there are additional massless exotics. For the cases in which the $U(1)^{\prime}$ is broken at the electroweak scale, the exotic fermions remain light compared to the electroweak scale, which is excluded by experiment. This feature indicates a general flaw of this type of models.

Trilinear terms. Nevertheless, we proceed and analyze the effective trilinear couplings. For each flat direction we determine all such couplings (to all orders in the VEV's of fields in the flat direction) in the observable sector of the theory, i.e., the SM and $U(1)^{\prime}$ sector of the theory. (In addition we include terms involving the hidden sector fields that play a role in the renormalization group analysis of the symmetry breaking scenarios, a topic of a subsequent paper [27].)

We then discuss the implications of these couplings. The predictions (from $\alpha_{s}$ ) for the electroweak gauge couplings are presented. At the level of the trilinear superpotential, we consider the fermion masses and textures, baryon- and lepton-number violating couplings, $R$-parity breaking terms and the absence of a stable lightest supersymmetric particle (LSP), and the occurrence of the non-canonical ("half") $\mu$-term. We identify types of symmetry breaking scenarios for $U(1)^{\prime}$, one at the electroweak scale [28-32] and another

\footnotetext{
${ }^{1}$ However, nonrenormalizable terms competitive in strength are also present in the original superpotential, as well as generated in a number of other ways, such as via the decoupling of heavy states [25], a nonminimal Kähler potential, and the corrections to the Kähler potential due to the large VEV's.
} 
one at an intermediate scale [28,33], and discuss the family non-universality of $Z^{\prime}$ couplings.

While we calculated the mass spectrum and the effective trilinear terms (in the observable sector) for all the flat directions (classified in [17]), we illustrate the techniques and present the detailed analysis along the steps discussed above for two representative flat directions.

In a subsequent paper [27] we plan to carry out further the phenomenological consequences by introducing soft supersymmetry breaking mass parameters; we shall analyze specific SM and $U(1)^{\prime}$ symmetry breaking patterns, consistent with experiment, and the particle mass spectrum at the electroweak scale.

The paper is structured as follows. In Sec. II, we review the flat direction analysis for this model given in [17], and present our techniques to compute the effective renormalizable superpotential for each flat direction considered. In Secs. III and IV the two respective representative examples are analyzed in detail. Conclusions and a discussion of further investigations are given in Sec. V.

\section{FLAT DIRECTIONS AND EFFECTIVE COUPLINGS}

The model we choose to analyze is model 5 of [11] (CHL5), which has the gauge group

$$
\begin{aligned}
& \left\{S U(3)_{C} \times S U(2)_{L}\right\}_{\mathrm{obs}} \times\left\{S U(4)_{2} \times S U(2)_{2}\right\}_{\mathrm{hid}} \\
& \times U(1)_{A} \times U(1)^{6} .
\end{aligned}
$$

In addition to the MSSM fields, the particle content includes the additional chiral superfields:

$$
\begin{aligned}
& 6(1,2,1,1)+(3,1,1,1)+(\overline{3}, 1,1,1)+4(1,2,1,2)+2(1,1,4,1) \\
& +10(1,1, \overline{4}, 1)+8(1,1,1,2)+5(1,1,4,2)+(1,1, \overline{4}, 2) \\
& +8(1,1,6,1)+3(1,1,1,3)+42(1,1,1,1)
\end{aligned}
$$

where the representation under $\left(S U(3)_{C}, S U(2)_{L}\right.$, $\left.S U(4)_{2}, S U(2)_{2}\right)$ is indicated. The complete list of fields with their $U(1)$ charges are presented in Tables $\mathrm{I}(\mathrm{a})-\mathrm{I}(\mathrm{c})$. $Q, u$, and $d$ denote quark candidates (doublets or singlets), with $\mathcal{D}$ reserved for an exotic quark singlet. $h$ generically denote Higgs or lepton doublet candidates and $e$ represents possible lepton singlet candidates, while $\varphi$ is left for other singlets. Capital letters are reserved for fields in non-trivial representations of the hidden sector non-Abelian groups.

The SM hypercharge is determined as a linear combination of the non-anomalous $U(1)$ 's, subject to basic phenomenological criteria (see [18] for more details). We require three families of quarks and leptons, as well as at least two candidate electroweak Higgs doublets, with conventional hypercharges. We also require grouping of all fields which are charged under $U(1)_{e m}$ and/or transform under $S U(3)_{C}$ into mirror pairs; this is a prerequisite to have the possibility of avoiding exactly massless charged and/or colored particles in the theory. In this model, these criteria lead to the unique hypercharge definition (first presented in [11])

$$
Y=\frac{1}{96}\left(-8 Q_{2}-3 Q_{3}-8 Q_{4}-Q_{5}+Q_{6}\right)
$$

[normalized to give $Y$ (quark doublet) $=1 / 6$ ], with KaćMoody level $k_{Y}=\frac{11}{3}$. (We calculate $k_{Y}$ using the universal Green-Schwarz relations; for more details, see [18].)

\section{A. Analysis of flat directions}

The presence of the anomalous $U(1)$ leads to the generation of a nonzero FI term $\xi$ in the corresponding $D$-term at genus $1[19,20]$ (at genus 2 for the dilaton tadpole [21]) in string theory with

$$
\xi=\frac{g_{\text {string }}^{2} M_{P}^{2}}{192 \pi^{2}} \operatorname{Tr} Q_{A},
$$

in which $g_{\text {string }}$ is related to the gauge coupling $g$ by the relation $g_{\text {string }}=g / \sqrt{2}[12]$ ( $g$ is normalized according to the standard [grand unified theory (GUT)] conventions, i.e., $\operatorname{Tr} T_{a} T_{b}=\delta_{a b} / 2$ for the generators of the fundamental representation of $S U(N))$ and $M_{P}$ is the reduced Planck mass, such that $M_{P}=M_{P l} / \sqrt{8 \pi}$, with $M_{P l} \sim 1.2 \times 10^{19} \mathrm{GeV}$.

The FI $D$-term triggers the scalar components $\varphi_{i}$ of certain chiral superfields $\Phi_{i}$ to acquire VEV's in such a way that $D$ - and $F$-flatness conditions are satisfied [19,22]. In principle, the fields which acquire VEV's may be those with nontrivial representations under the observable sector or the non-Abelian hidden sector. For simplicity, we restrict our consideration to the non-Abelian singlet fields of the model, and select only those fields with zero hypercharge to preserve the SM gauge group at the string scale. The $D$ - and $F$-flatness conditions for these fields are given by

$$
\begin{aligned}
& D_{\mathrm{A}}=\sum_{i} Q_{i}^{(A)}\left|\varphi_{i}\right|^{2}+\xi=0 \\
& D_{a}=\sum_{i} Q_{i}^{(a)}\left|\varphi_{i}\right|^{2}=0 \\
& F_{i}=\frac{\partial W}{\partial \Phi_{i}}=0 ; \quad W=0 .
\end{aligned}
$$

We list the non-Abelian singlets of the model with their $U(1)$ charges in Table I.

In a previous paper [17], we presented techniques to classify the $D$ - and $F$-flat directions of a general perturbative heterotic string model with an anomalous $U(1)$, and illustrated the method by applying it to this model. We summarize the method and repeat the conclusions here for the sake of completeness, and refer the reader to [17] for more details.

First, the $D$-flat directions associated with the nonanomalous $U(1)$ 's are determined, by making use of the one-to-one correspondence of $D$-flat directions with holomorphic gauge-invariant monomials (HIM's) of chiral superfields [34-36], constructed from the non-Abelian singlets. We construct the set of all one-dimensional HIM's, which 
TABLE I. (a) List of non-Abelian non-singlet observable sector fields in the model with their charges under the $U(1)$ gauge groups, hypercharge as defined in Eq. (3), and $U(1)^{\prime}$ as defined in Eq. (12). The second column introduces the notation used throughout this paper, and the third column the translation to the notation used in [11] (CHL). (b) List of non-Abelian non-singlet hidden sector fields in the model with their charges under the $U(1)$ gauge groups, hypercharge as defined in Eq. (3), and $U(1)^{\prime}$ as defined in Eq. (12). [We largely follow the notation of [11] (CHL).] (c): List of non-Abelian singlet fields in the model with their charges under the $U(1)$ gauge groups, hypercharge as defined in Eq. (3), and $U(1)^{\prime}$ as defined in Eq. (12). The first column gives the notation used throughout this paper, the second column the translation to the notation used in [11] (CHL), and the third column the translation to the notation used in [17].

(a)

$\left(S U(3)_{C}, S U(2)_{L}\right.$ $\left.S U(4)_{2}, S U(2)_{2}\right)$

\begin{tabular}{|c|c|c|c|c|c|c|c|c|c|c|c|}
\hline \multirow[t]{3}{*}{$(3,2,1,1)$} & $Q_{a}$ & $Q_{1}$ & -2 & 0 & 8 & -2 & -8 & 16 & -16 & 1 & 68 \\
\hline & $Q_{b}$ & $Q_{2}$ & 2 & 0 & 8 & -2 & -8 & 16 & -16 & 1 & 68 \\
\hline & $Q_{c}$ & $Q_{3}$ & 0 & -2 & 0 & -2 & 20 & 4 & -12 & 1 & -71 \\
\hline \multirow[t]{7}{*}{$(\overline{3}, 1,1,1)$} & $u_{a}^{c}$ & $u_{1}^{c}$ & 2 & 0 & 0 & 6 & 0 & -16 & -16 & -4 & 6 \\
\hline & $u_{b}^{c}$ & $u_{2}^{c}$ & -2 & 0 & 0 & 6 & 0 & -16 & -16 & -4 & 6 \\
\hline & $u_{c}^{c}$ & $u_{3}^{c}$ & 0 & 6 & -8 & 2 & -4 & -28 & -12 & -4 & -133 \\
\hline & $d_{a}^{c}$ & $d_{1}^{c}$ & 0 & -6 & -8 & 2 & -4 & -28 & -12 & 2 & -3 \\
\hline & $d_{b}^{c}$ & $d_{2}^{c}$ & 0 & 0 & 0 & 0 & -12 & 20 & 4 & 2 & 136 \\
\hline & $d_{c}^{c}$ & $d_{3}^{c}$ & 0 & 2 & -8 & -2 & 0 & 8 & 8 & 2 & -3 \\
\hline & $d_{d}^{c}$ & $\bar{t}$ & 0 & 2 & -8 & -2 & 0 & 8 & 8 & 2 & -3 \\
\hline \multirow[t]{11}{*}{$(1,2,1,1)$} & $\bar{h}_{a}$ & $\bar{h}_{1}$ & 0 & 0 & -8 & -4 & -4 & -12 & 4 & 3 & -74 \\
\hline & $\bar{h}_{b}$ & $\bar{h}_{2}$ & 0 & -2 & -16 & 2 & 0 & 0 & 0 & 3 & 65 \\
\hline & $\bar{h}_{c}$ & $\bar{h}_{3}$ & 0 & -4 & 8 & 0 & -16 & 24 & 24 & 3 & 204 \\
\hline & $\bar{h}_{d}$ & $\bar{h}_{4}$ & 0 & -2 & 0 & -2 & 8 & 24 & -8 & 3 & 65 \\
\hline & $h_{a}$ & $h_{1}$ & 0 & 0 & 8 & 4 & 4 & 12 & -4 & -3 & 74 \\
\hline & $h_{b}$ & $h_{2}$ & 0 & 2 & 0 & 2 & 16 & 0 & 0 & -3 & -65 \\
\hline & $h_{c}$ & $h_{3}$ & 0 & 2 & 0 & 2 & -8 & -24 & 8 & -3 & -65 \\
\hline & $h_{d}$ & $h_{4}$ & 0 & 2 & 16 & -2 & 0 & 0 & 0 & -3 & -65 \\
\hline & $h_{e}$ & $L_{1}$ & 0 & 4 & -8 & 0 & 16 & -24 & -24 & -3 & -204 \\
\hline & $h_{f}$ & $L_{2}$ & 0 & 2 & 16 & -2 & -12 & -12 & -28 & -3 & -65 \\
\hline & $h_{g}$ & $L_{3}$ & 0 & 2 & 0 & 2 & 16 & 0 & 0 & -3 & -65 \\
\hline$(3,1,1,1)$ & $\mathcal{D}_{a}$ & $t$ & 0 & 0 & 0 & 0 & 12 & -20 & -4 & -2 & -136 \\
\hline \multicolumn{12}{|c|}{ (b) } \\
\hline $\begin{array}{l}\left(S U(3)_{C}, S U(2)_{L},\right. \\
\left.S U(4)_{2}, S U(2)_{2}\right)\end{array}$ & & & $Q_{1}$ & $Q_{2}$ & $Q_{3}$ & $Q_{4}$ & $Q_{5}$ & $Q_{6}$ & $Q_{A}$ & $6 Q_{Y}$ & $100 Q_{Y^{\prime}}$ \\
\hline$(1,2,1,2)$ & $D_{1-4}$ & & 0 & 0 & -8 & 2 & -4 & -12 & 4 & 0 & 0 \\
\hline$(1,1,4,1)$ & $F_{1,2}$ & & 0 & 0 & 12 & 0 & 12 & 0 & -16 & -3 & -65 \\
\hline \multirow[t]{4}{*}{$(1,1, \overline{4}, 1)$} & $\bar{F}_{1,2}$ & & 0 & 0 & 4 & -4 & -4 & 24 & 8 & 3 & 65 \\
\hline & $\bar{F}_{3-6}$ & & 0 & 0 & -12 & 0 & 0 & 12 & -20 & 3 & 65 \\
\hline & $\bar{F}_{7,8}$ & & 2 & 0 & 4 & 2 & 8 & -12 & -12 & -3 & -65 \\
\hline & $\bar{F}_{9,10}$ & & -2 & 0 & 4 & 2 & 8 & -12 & -12 & -3 & -65 \\
\hline \multirow[t]{4}{*}{$(1,1,1,2)$} & $H_{1,2}$ & & 0 & -2 & 8 & -4 & -12 & 12 & -4 & 3 & 65 \\
\hline & $H_{3,4}$ & & 0 & -4 & 0 & 2 & -8 & 24 & -8 & 3 & 204 \\
\hline & $H_{5,7}$ & & 0 & 2 & -8 & 4 & 12 & -12 & 4 & -3 & -65 \\
\hline & $H_{6,8}$ & & 0 & 0 & 16 & 2 & -16 & 0 & 0 & -3 & 74 \\
\hline \multirow[t]{3}{*}{$(1,1,4,2)$} & $E_{1,2}$ & & 0 & 0 & -4 & -2 & 4 & -24 & -8 & 0 & -139 \\
\hline & $E_{3}$ & & 0 & -2 & 4 & 0 & 4 & 0 & 16 & 0 & 0 \\
\hline & $E_{4,5}$ & & 0 & -2 & 4 & 0 & -8 & -12 & -12 & 0 & 0 \\
\hline$(1,1, \overline{4}, 2)$ & $\bar{E}_{1}$ & & 0 & 2 & -4 & 0 & -4 & 0 & -16 & 0 & 0 \\
\hline \multirow[t]{2}{*}{$(1,1,6,1)$} & $S_{1}$ & & 2 & 0 & -8 & 2 & -4 & -12 & 4 & 0 & 0 \\
\hline & $S_{2}$ & & 0 & -4 & 8 & 0 & -4 & -12 & 4 & 0 & 0 \\
\hline
\end{tabular}


TABLE I. (Continued.)

\begin{tabular}{|c|c|c|c|c|c|c|c|c|c|c|}
\hline & & & & & & & & & & \\
\hline $\begin{array}{l}\left(S U(3)_{C}\right. \\
S U(4)_{2},\end{array}$ & & $Q_{1}$ & $Q_{2}$ & $Q_{3}$ & $Q_{4}$ & $Q_{5}$ & $Q_{6}$ & $Q_{A}$ & $6 Q_{Y}$ & $100 Q_{Y^{\prime}}$ \\
\hline & $S_{3}$ & -2 & 0 & -8 & 2 & -4 & -12 & 4 & 0 & 0 \\
\hline & $S_{4}$ & 2 & 0 & 8 & -2 & 4 & 12 & -4 & 0 & 0 \\
\hline & $S_{5}$ & 0 & 4 & -8 & 0 & 4 & 12 & -4 & 0 & 0 \\
\hline & $S_{6,7}$ & 0 & 2 & 0 & 2 & -8 & 24 & -8 & 0 & 139 \\
\hline & $S_{8}$ & -2 & 0 & 8 & -2 & 4 & 12 & -4 & 0 & 0 \\
\hline$(1,1,1,3)$ & $T_{1}$ & 2 & 4 & 0 & -2 & 8 & 24 & -8 & 0 & 0 \\
\hline & $T_{2}$ & 0 & -2 & 8 & 2 & 12 & 36 & -12 & 0 & 139 \\
\hline & $T_{3}$ & -2 & 4 & 0 & -2 & 8 & 24 & -8 & 0 & 0 \\
\hline
\end{tabular}

(c)

$\begin{array}{lllllllll}Q_{1} & Q_{2} & Q_{3} & Q_{4} & Q_{5} & Q_{6} & Q_{A} & 6 Q_{Y} & 100 Q_{Y^{\prime}}\end{array}$

\begin{tabular}{|c|c|c|c|c|c|c|c|c|c|c|c|}
\hline$e_{a, c}^{c}$ & $e_{1,3}^{c}$ & $S_{25,25^{\prime}}$ & 0 & -2 & -8 & -6 & 4 & 12 & -4 & 6 & -9 \\
\hline$e_{b}^{c}$ & $e_{2}^{c}$ & $S_{10}$ & 4 & -2 & -24 & -2 & -4 & -12 & 4 & 6 & -9 \\
\hline$e_{d, g}^{c}$ & $s_{1,4}$ & $S_{21,21^{\prime}}$ & 0 & -4 & 0 & -4 & -8 & 24 & -8 & 6 & 130 \\
\hline$e_{e}^{c}$ & $s_{2}$ & $S_{13}$ & 2 & 0 & -16 & -2 & -32 & 0 & 0 & 6 & 130 \\
\hline$e_{f}^{c}$ & $s_{3}$ & $\bar{S}_{2}$ & 0 & -4 & -16 & 0 & 8 & 24 & -8 & 6 & 130 \\
\hline$e_{h}^{c}$ & $s_{5}$ & $S_{17}$ & -2 & 0 & -16 & -2 & -32 & 0 & 0 & 6 & 130 \\
\hline$e_{i}^{c}$ & $s_{6}$ & $S_{9}$ & -4 & -2 & -24 & -2 & -4 & -12 & 4 & 6 & -9 \\
\hline$e_{a, b}$ & $\bar{s}_{1,2}$ & $S_{16,16^{\prime}}$ & 0 & 4 & 16 & 0 & 16 & 0 & 0 & -6 & -130 \\
\hline$e_{c}$ & $\bar{s}_{3}$ & $S_{2}$ & 0 & 4 & 16 & 0 & -8 & -24 & 8 & -6 & -130 \\
\hline$e_{d, e}$ & $\bar{s}_{4,5}$ & $S_{24,24^{\prime}}$ & 0 & 2 & 24 & 2 & 4 & 12 & -4 & -6 & 9 \\
\hline$e_{f}$ & $\bar{s}_{6}$ & $S_{7}$ & 0 & -2 & 8 & 2 & 12 & -60 & 20 & -6 & -269 \\
\hline$\varphi_{1}$ & $\varphi_{1}$ & $S_{4}$ & 4 & 0 & 0 & 0 & 0 & 0 & 0 & 0 & 0 \\
\hline$\varphi_{2,3}$ & $\varphi_{2,3}$ & $S_{5,5^{\prime}}$ & 2 & 4 & 0 & -2 & -16 & 0 & 0 & 0 & 0 \\
\hline$\varphi_{4,5}$ & $\varphi_{4,5}$ & $S_{14,14^{\prime}}$ & 2 & -4 & 16 & -2 & 0 & 0 & 0 & 0 & 0 \\
\hline$\varphi_{6,7}$ & $\varphi_{6,7}$ & $S_{6,6^{\prime}}$ & 2 & -4 & 0 & 2 & 16 & 0 & 0 & 0 & 0 \\
\hline$\varphi_{8,9}$ & $\varphi_{8,9}$ & $S_{15,15^{\prime}}$ & 0 & 0 & -16 & 4 & 16 & 0 & 0 & 0 & 0 \\
\hline$\varphi_{10,11}$ & $\varphi_{10,11}$ & $\bar{S}_{6,6^{\prime}}$ & -2 & 4 & 0 & -2 & -16 & 0 & 0 & 0 & 0 \\
\hline$\varphi_{12,13}$ & $\varphi_{12,13}$ & $S_{18,18^{\prime}}$ & -2 & -4 & 16 & -2 & 0 & 0 & 0 & 0 & 0 \\
\hline$\varphi_{14,15}$ & $\varphi_{14,15}$ & $\bar{S}_{5,5^{\prime}}$ & -2 & -4 & 0 & 2 & 16 & 0 & 0 & 0 & 0 \\
\hline$\varphi_{16}$ & $\varphi_{16}$ & $\bar{S}_{4}$ & -4 & 0 & 0 & 0 & 0 & 0 & 0 & 0 & 0 \\
\hline$\varphi_{17}$ & $\chi_{1}$ & $S_{22}$ & 2 & 4 & 0 & -2 & 8 & 24 & -8 & 0 & 0 \\
\hline$\varphi_{18,19}$ & $\chi_{2,3}$ & $S_{11,11^{\prime}}$ & 0 & 2 & 8 & -6 & -4 & -12 & 4 & 0 & -139 \\
\hline$\varphi_{20,21}$ & $\chi_{4,5}$ & $S_{19,19^{\prime}}$ & 0 & 2 & -8 & -2 & 0 & -24 & -24 & 0 & -139 \\
\hline$\varphi_{22}$ & $\chi_{6}$ & $S_{12}$ & 0 & 2 & -24 & 2 & 28 & -12 & 4 & 0 & -139 \\
\hline$\varphi_{23}$ & $\chi_{7}$ & $\bar{S}_{3}$ & 0 & 0 & 16 & -4 & 8 & 24 & -8 & 0 & 0 \\
\hline$\varphi_{24}$ & $\chi_{8}$ & $\bar{S}_{1}$ & 0 & 0 & 0 & 0 & -12 & -12 & -28 & 0 & 0 \\
\hline$\varphi_{25}$ & $\chi_{9}$ & $S_{20}$ & 0 & -2 & 8 & 2 & 12 & 36 & -12 & 0 & 139 \\
\hline$\varphi_{26}$ & $\chi_{10}$ & $S_{23}$ & -2 & 4 & 0 & -2 & 8 & 24 & -8 & 0 & 0 \\
\hline$\varphi_{27}$ & $\chi_{11}$ & $S_{1}$ & 0 & 0 & 0 & 0 & 12 & 12 & 28 & 0 & 0 \\
\hline$\varphi_{28,29}$ & $\chi_{12,13}$ & $S_{8,8^{\prime}}$ & 0 & 0 & 0 & 0 & -24 & -24 & 8 & 0 & 0 \\
\hline$\varphi_{30}$ & $\chi_{14}$ & $S_{3}$ & 0 & 0 & -16 & 4 & -8 & -24 & 8 & 0 & 0 \\
\hline
\end{tabular}

we refer to as the superbasis. The complete set of $D$-flat directions under the non-anomalous $U(1)$ 's can be obtained by multiplying the elements of the superbasis. The elements of the superbasis with anomalous charge opposite in sign to the FI term $\xi$ are flat with respect to the $D$-term of the anomalous $U(1)$. These superbasis elements, which we denote as $\left\{P_{\alpha}\right\}$, are the building blocks for the $D$-flat directions of the model.

We presented the superbasis in [17], and showed that there are five such classes of $D_{A}$-flat elements: 


$$
\begin{aligned}
& P_{1}=\left\langle\varphi_{28}, \varphi_{27}^{2}\right\rangle, \\
& P_{2}=\left\langle\varphi_{4}, \varphi_{10}, \varphi_{30}, \varphi_{27}^{2}\right\rangle, \\
& P_{3}=\left\langle\varphi_{12}, \varphi_{2}, \varphi_{30}, \varphi_{27}^{2}\right\rangle, \\
& P_{4}=\left\langle\varphi_{4}, \varphi_{2}, \varphi_{16}, \varphi_{30}, \varphi_{27}^{2}\right\rangle, \\
& P_{5}=\left\langle\varphi_{12}, \varphi_{10}, \varphi_{1}, \varphi_{30}, \varphi_{27}^{2}\right\rangle .
\end{aligned}
$$

To these, one should add similar monomials obtained by replacing some field by its copy $\left(\varphi_{2} \rightarrow \varphi_{3}, \varphi_{4} \rightarrow \varphi_{5}, \varphi_{10}\right.$ $\left.\rightarrow \varphi_{11}, \varphi_{12} \rightarrow \varphi_{13}, \varphi_{28} \rightarrow \varphi_{29}\right)$. Therefore, every $D_{A}$-flat direction can be obtained from the set $\left\{P_{\alpha}\right\}$ by

$$
P=P_{\alpha} N
$$

with $N$ some HIM (not necessarily with $Q_{A}>0$ ).

To address the $F$-flatness of the $D$-flat directions, we note that there are two classes of terms in the superpotential that can lift a general $D$-flat direction $P$. First, there can be terms which are formed only of the fields in the flat direction $P$ :

$$
W_{A} \sim\left(\Pi_{i \in P} \Phi_{i}\right)^{n}
$$

in which the coefficients (which depend on inverse powers of $\left.M_{P l}\right)$ are not displayed explicitly. The flat direction $P$ will be said to be type-A if such an invariant is allowed by gauge symmetries. If such an invariant exists, there are an infinite number of terms which can lift the flat direction, because this invariant can appear to any power in the superpotential. The type-A directions will remain $F$-flat only if string selection rules (e.g. $R$ parities) conspire to forbid the infinite number of $W_{A}$ terms, which is difficult to prove in general.

The other class of terms are of the form

$$
W_{B} \sim \Psi\left(\Pi_{i \in P} \Phi_{i}\right),
$$

with $\Psi \notin P$. A flat direction will be denoted as type-B if gauge invariance only allows $W_{B}$ terms. In contrast to the case with the $W_{A}$ terms, gauge invariance constrains the number of $W_{B}$ terms which can exist to a finite number. By doing a string calculation of the superpotential to a finite order, the presence of these terms can be checked explicitly, and if such terms are absent, the flat direction is proved to be $F$-flat to all orders in the nonrenormalizable superpotential. We take a conservative approach by restricting our consideration to the type-B directions which can be proved to be $F$-flat to all orders. In doing so, we are of course neglecting certain type-A directions that may be $F$-flat due to "string selection rules" of the model.

It is straightforward to show [17] that type-B directions are formed only from carefully combining the $P_{\alpha}$ 's. In this model, we found that $P_{4}$ and $P_{5}$ are not $F$-flat, and thus the $F$-flat directions are formed from $P_{1}, P_{2}$ and $P_{3}$ (and the primed versions of them involving copies of the fields). We present the complete list of $F$-flat directions in Table II.

This table demonstrates that there are a range of flat directions, which break different numbers of the nonanomalous $U(1)$ 's. We choose to analyze the $P_{2} P_{3}$ and
$P_{1} P_{2} P_{3}$ flat directions, which break the maximum number of $U(1)$ 's; these directions all leave an additional $U(1)^{\prime}$ as well as $U(1)_{Y}$ unbroken. The unbroken $U(1)^{\prime}$ is given by

$$
Y^{\prime}=\frac{1}{1200}\left(-130 Q_{2}-14 Q_{3}+148 Q_{4}-51 Q_{5}+51 Q_{6}\right),
$$

with $k_{Y^{\prime}}=4167 / 250 \simeq 16.67$.

The $D$-term constraints for the VEV's of the fields in the most general $P_{1} P_{2} P_{3}$ direction yield the relations

$$
\begin{gathered}
\left|\varphi_{27}\right|^{2}=2 x^{2}, \quad\left|\varphi_{28(29)}\right|^{2}=x^{2}-\left|\psi_{1}\right|^{2}, \\
\left|\varphi_{30}\right|^{2}=\left|\psi_{1}\right|^{2}, \quad\left|\varphi_{4(5)}\right|^{2}=\left|\psi_{2}\right|^{2}, \\
\left|\varphi_{2(3)}\right|^{2}=\left|\psi_{1}\right|^{2}-\left|\psi_{2}\right|^{2}, \quad\left|\varphi_{12(13)}\right|^{2}=\left|\psi_{1}\right|^{2}-\left|\psi_{2}\right|^{2}, \\
\left|\varphi_{10(11)}\right|^{2}=\left|\psi_{2}\right|^{2},
\end{gathered}
$$

with

$$
x^{2}=-\frac{\xi}{64}
$$

In this model $\operatorname{Tr} Q_{A}=-1536$, and $x=0.013 M_{P l}$. Here $\left|\psi_{1,2}\right|$ are free VEV's of the moduli space, subject to the restrictions that

$$
x^{2} \geqslant\left|\psi_{1}\right|^{2} \geqslant\left|\psi_{2}\right|^{2},
$$

to ensure the positivity of the VEV squares. Simpler flat directions are recovered by setting the free VEV's to particular values; for example, setting $\left|\psi_{1}\right|^{2}=\left|\psi_{2}\right|^{2}=0$ yields the solution for the VEV's of $P_{1}$. Therefore, there can be an enhanced number of $U(1)$ 's at particular points in the moduli space. The $P_{2} P_{3}$ directions are obtained by setting $\left|\psi_{1}\right|^{2}=x^{2}$, which gives $\left|\varphi_{28(29)}\right|^{2}=0$.

In some cases, a judicious choice of the copies of the fields allows for $F$-flatness without imposing any constraints on the free VEV's. However, other possible flat directions can be formed by imposing constraints on the free VEV's in such a way as to cancel contributions from different $F$-terms, i.e., of the type $\varphi_{9}\left(\varphi_{4} \varphi_{10(11)}+\varphi_{2(3)} \varphi_{12}\right)$ [17]. For example, the directions denoted by $\left.\right|_{F}$ are obtained by imposing $\left|\psi_{1}\right|^{2}=2\left|\psi_{2}\right|^{2}$ and the $\pi$ phase difference between VEV's of the $\varphi_{4} \varphi_{10(11)}$ and $\varphi_{2(3)} \varphi_{12}$ terms [17]. ${ }^{2}$ The complete list of all such flat directions is given in Table II.

\footnotetext{
${ }^{2}$ Throughout the paper we assume that these VEV's are real. For the model discussed the introduction of complex phases for these VEV's can be absorbed into the redefinition of the remaining fields in the effective superpotential, and thus it does not affect the physics at the level of the effective bilinear and trilinear terms.
} 
TABLE II. The explicit list of type-B $D$-flat directions that are $F$-flat to all orders for the CHL5 model. (This table expands the compact presentation of these flat directions given in [17].) The dimension of the direction, after cancellation of the Fayet-Iliopoulos term, is indicated in the second column. The third column gives the number (out of 6 ) of non-anomalous $U(1)$ 's broken along the flat direction.

\begin{tabular}{|c|c|c|}
\hline Flat direction & Dim. & No. $U(1)$ 's \\
\hline$P_{1}=\left\langle\varphi_{28}, \varphi_{27}^{2}\right\rangle$ & 0 & 1 \\
\hline$P_{1}^{\prime}=\left\langle\varphi_{29}, \varphi_{27}^{2}\right\rangle$ & 0 & 1 \\
\hline$P_{2}^{\prime}=\left\langle\varphi_{5}, \varphi_{10}, \varphi_{30}, \varphi_{27}^{2}\right\rangle$ & 0 & 3 \\
\hline$P_{2}^{\prime \prime}=\left\langle\varphi_{5}, \varphi_{11}, \varphi_{30}, \varphi_{27}^{2}\right\rangle$ & 0 & 3 \\
\hline$P_{3}^{\prime}=\left\langle\varphi_{13}, \varphi_{2}, \varphi_{30}, \varphi_{27}^{2}\right\rangle$ & 0 & 3 \\
\hline$P_{3}^{\prime \prime}=\left\langle\varphi_{13}, \varphi_{3}, \varphi_{30}, \varphi_{27}^{2}\right\rangle$ & 0 & 3 \\
\hline$\left.P_{2} P_{3}\right|_{F}=\left.\left\langle\varphi_{12}, \varphi_{10}, \varphi_{4}, \varphi_{2}, \varphi_{30}^{2}, \varphi_{27}^{4}\right\rangle\right|_{F}$ & 0 & 4 \\
\hline$\left.P_{2}^{\prime \prime \prime} P_{3}^{\prime \prime \prime}\right|_{F}=\left.\left\langle\varphi_{12}, \varphi_{11}, \varphi_{4}, \varphi_{3}, \varphi_{30}^{2}, \varphi_{27}^{4}\right\rangle\right|_{F}$ & 0 & 4 \\
\hline$P_{1} P_{1}^{\prime}=\left\langle\varphi_{28}, \varphi_{29}, \varphi_{27}^{4}\right\rangle$ & 1 & 1 \\
\hline$P_{2}^{\prime} P_{2}^{\prime \prime}=\left\langle\varphi_{5}^{2}, \varphi_{10}, \varphi_{11}, \varphi_{30}^{2}, \varphi_{27}^{4}\right\rangle$ & 1 & 3 \\
\hline$P_{3}^{\prime} P_{3}^{\prime \prime}=\left\langle\varphi_{2}^{2}, \varphi_{12}, \varphi_{13}, \varphi_{30}^{2}, \varphi_{27}^{4}\right\rangle$ & 1 & 3 \\
\hline$P_{1} P_{2}^{\prime}=\left\langle\varphi_{28}, \varphi_{5}, \varphi_{10}, \varphi_{30}, \varphi_{27}^{4}\right\rangle$ & 1 & 3 \\
\hline$P_{1} P_{2}^{\prime \prime}=\left\langle\varphi_{28}, \varphi_{5}, \varphi_{11}, \varphi_{30}, \varphi_{27}^{4}\right\rangle$ & 1 & 3 \\
\hline$P_{1}^{\prime} P_{2}^{\prime}=\left\langle\varphi_{29}, \varphi_{5}, \varphi_{10}, \varphi_{30}, \varphi_{27}^{4}\right\rangle$ & 1 & 3 \\
\hline$P_{1}^{\prime} P_{2}^{\prime \prime}=\left\langle\varphi_{29}, \varphi_{5}, \varphi_{11}, \varphi_{30}, \varphi_{27}^{4}\right\rangle$ & 1 & 3 \\
\hline$P_{1} P_{3}^{\prime}=\left\langle\varphi_{28}, \varphi_{2}, \varphi_{13}, \varphi_{30}, \varphi_{27}^{4}\right\rangle$ & 1 & 3 \\
\hline$P_{1} P_{3}^{\prime \prime}=\left\langle\varphi_{28}, \varphi_{3}, \varphi_{13}, \varphi_{30}, \varphi_{27}^{4}\right\rangle$ & 1 & 3 \\
\hline$P_{1}^{\prime} P_{3}^{\prime}=\left\langle\varphi_{29}, \varphi_{2}, \varphi_{13}, \varphi_{30}, \varphi_{27}^{4}\right\rangle$ & 1 & 3 \\
\hline$P_{1}^{\prime} P_{3}^{\prime \prime}=\left\langle\varphi_{29}, \varphi_{3}, \varphi_{13}, \varphi_{30}, \varphi_{27}^{4}\right\rangle$ & 1 & 3 \\
\hline$P_{2}^{\prime} P_{3}^{\prime}=\left\langle\varphi_{2}, \varphi_{5}, \varphi_{10}, \varphi_{13}, \varphi_{30}^{2}, \varphi_{27}^{4}\right\rangle$ & 1 & 4 \\
\hline$P_{2}^{\prime} P_{3}^{\prime \prime}=\left\langle\varphi_{3}, \varphi_{5}, \varphi_{10}, \varphi_{13}, \varphi_{30}^{2}, \varphi_{27}^{4}\right\rangle$ & 1 & 4 \\
\hline$P_{2}^{\prime \prime} P_{3}^{\prime}=\left\langle\varphi_{2}, \varphi_{5}, \varphi_{11}, \varphi_{13}, \varphi_{30}^{2}, \varphi_{27}^{4}\right\rangle$ & 1 & 4 \\
\hline$P_{2}^{\prime \prime} P_{3}^{\prime \prime}=\left\langle\varphi_{3}, \varphi_{5}, \varphi_{11}, \varphi_{13}, \varphi_{30}^{2}, \varphi_{27}^{4}\right\rangle$ & 1 & 4 \\
\hline$\left.P_{1} P_{2} P_{3}\right|_{F}=\left.\left\langle\varphi_{12}, \varphi_{10}, \varphi_{4}, \varphi_{2}, \varphi_{28}, \varphi_{30}^{2}, \varphi_{27}^{6}\right\rangle\right|_{F}$ & 1 & 4 \\
\hline$\left.P_{1}^{\prime} P_{2} P_{3}\right|_{F}=\left.\left\langle\varphi_{12}, \varphi_{10}, \varphi_{4}, \varphi_{2}, \varphi_{29}, \varphi_{30}^{2}, \varphi_{27}^{6}\right\rangle\right|_{F}$ & 1 & 4 \\
\hline$\left.P_{1} P_{2}^{\prime \prime \prime} P_{3}^{\prime \prime \prime}\right|_{F}=\left.\left\langle\varphi_{12}, \varphi_{11}, \varphi_{4}, \varphi_{3}, \varphi_{28}, \varphi_{30}^{2}, \varphi_{27}^{6}\right\rangle\right|_{F}$ & 1 & 4 \\
\hline$\left.P_{1}^{\prime} P_{2}^{\prime \prime \prime} P_{3}^{\prime \prime \prime}\right|_{F}=\left.\left\langle\varphi_{12}, \varphi_{11}, \varphi_{4}, \varphi_{3}, \varphi_{29}, \varphi_{30}^{2}, \varphi_{27}^{6}\right\rangle\right|_{F}$ & 1 & 4 \\
\hline$P_{1} P_{2}^{\prime} P_{3}^{\prime}=\left\langle\varphi_{13}, \varphi_{10}, \varphi_{5}, \varphi_{2}, \varphi_{28}, \varphi_{30}^{2}, \varphi_{27}^{6}\right\rangle$ & 2 & 4 \\
\hline$P_{1}^{\prime} P_{2}^{\prime} P_{3}^{\prime}=\left\langle\varphi_{13}, \varphi_{10}, \varphi_{5}, \varphi_{2}, \varphi_{29}, \varphi_{30}^{2}, \varphi_{27}^{6}\right\rangle$ & 2 & 4 \\
\hline$P_{1} P_{2}^{\prime} P_{3}^{\prime \prime}=\left\langle\varphi_{13}, \varphi_{10}, \varphi_{5}, \varphi_{3}, \varphi_{28}, \varphi_{30}^{2}, \varphi_{27}^{6}\right\rangle$ & 2 & 4 \\
\hline$P_{1}^{\prime} P_{2}^{\prime} P_{3}^{\prime \prime}=\left\langle\varphi_{13}, \varphi_{10}, \varphi_{5}, \varphi_{3}, \varphi_{29}, \varphi_{30}^{2}, \varphi_{27}^{6}\right\rangle$ & 2 & 4 \\
\hline$P_{1} P_{2}^{\prime \prime} P_{3}^{\prime}=\left\langle\varphi_{13}, \varphi_{11}, \varphi_{5}, \varphi_{2}, \varphi_{28}, \varphi_{30}^{2}, \varphi_{27}^{6}\right\rangle$ & 2 & 4 \\
\hline$P_{1}^{\prime} P_{2}^{\prime \prime} P_{3}^{\prime}=\left\langle\varphi_{13}, \varphi_{11}, \varphi_{5}, \varphi_{2}, \varphi_{29}, \varphi_{30}^{2}, \varphi_{27}^{6}\right\rangle$ & 2 & 4 \\
\hline$P_{1} P_{2}^{\prime \prime} P_{3}^{\prime \prime}=\left\langle\varphi_{13}, \varphi_{11}, \varphi_{5}, \varphi_{3}, \varphi_{28}, \varphi_{30}^{2}, \varphi_{27}^{6}\right\rangle$ & 2 & 4 \\
\hline$P_{1}^{\prime} P_{2}^{\prime \prime} P_{3}^{\prime \prime}=\left\langle\varphi_{13}, \varphi_{11}, \varphi_{5}, \varphi_{3}, \varphi_{29}, \varphi_{30}^{2}, \varphi_{27}^{6}\right\rangle$ & 2 & 4 \\
\hline
\end{tabular}

\section{B. Mass spectrum}

For each flat direction, effective mass terms for fields $\Psi_{i}, \Psi_{j}\left(\Psi_{i, j} \notin\left\{\Phi_{k}\right\}\right)$ may be generated by the coupling of these fields to the fields $\Phi_{i}$ in the flat direction, such that

$$
W \sim \Psi_{i} \Psi_{j}\left(\Pi_{k \in P} \Phi_{k}\right) .
$$

The fields with effective mass terms will acquire $F$-term string-scale masses and decouple from the low-energy theory. In cases in which $F$-flatness occurs via cancellations, other fields coupled linearly to the flat directions fields also get heavy masses (we will later study such a case).

In addition to these large masses induced by $F$-terms, $D$-terms can also make some (combination) of the fields related to the flat direction heavy (all other fields do not feel the presence of large VEVs in the $D$-terms because of the $D$-flatness conditions). Particular combinations of the real components of the fields entering the flat direction gain a 
mass of order $g \sqrt{\xi}$ (through $D$-terms) and become degenerate with the massive $U(1)$ gauge bosons, completing, along with a Dirac fermion (a neutralino), a massive vector multiplet. This is guaranteed by the fact that supersymmetry remains unbroken in the restabilized vacuum, so that the spectrum must arrange itself in supersymmetric multiplets. ${ }^{3}$ The imaginary parts of these fields become the longitudinal components of the massive $U(1)$ gauge bosons. (This scenario thus exhibits all the features of the Higgs mechanism in $N$ $=1$ supersymmetric theory.)

For directions which are flat due to cancellations of $F$-term contributions, some (complex) fields in the flat direction get masses of order "[Yukawa] $\times$ [field VEV]" and form, along with its superpartner, a massive chiral superfield. (As mentioned above, the fields with zero VEV's which couple linearly in these terms also acquire mass of the same order.) Remaining fields in the flat direction, including the real parts of the fields whose VEV is not fixed by the flatness conditions, as well as imaginary parts not removed by the Higgs mechanism, will stay massless and will appear in the low energy theory ${ }^{4}$ as massless chiral superfields (moduli).

For the flat directions of the type $\left.P_{2} P_{3}\right|_{F}$, which has all the VEV's fixed, all these (six) fields are heavy. Namely, the Higgs mechanism ensures that five imaginary components are Goldstone bosons, giving mass to the gauge bosons of five (including anomalous) $U(1)$ 's, and the accompanying five real components get mass, thus completing five massive vector supermultiplets. The remaining complex field gets a mass from the superpotential terms of the type $\varphi_{9}\left(\varphi_{4} \varphi_{10(11)}+\varphi_{2(3)} \varphi_{12}\right)$, i.e., the terms which require additional constraints on the VEV's in order to ensure $F$-flatness. Note that due to these terms $\varphi_{9}$ also acquires a mass.

For the flat directions of the type $P_{1} P_{2} P_{3}$, there are two free VEV parameters, since now an additional field participates in the vacuum restabilization and there are no constraints on the VEV's from $F$-flatness constraints (as opposed to the $\left.P_{1} P_{2}\right|_{F}$ flat directions), and the corresponding analysis shows that now there are two massless complex fields, which act as moduli in the space of restabilized vacua.

\section{Effective couplings}

The spectrum of the low-energy theory not only arranges itself in supersymmetric (SUSY) multiplets, but also the in-

\footnotetext{
${ }^{3}$ In more detail this works as follows. Using the matrix $m_{a i}$ $=\sqrt{2} g_{a} Q_{i}^{(a)}\left\langle\varphi_{i}\right\rangle$, the squared-mass matrix of $U(1)$ gauge bosons is $\mathrm{mm}^{T}$ while that of real scalars, coming from $D$-terms, is $m^{T} m$. It is a simple exercise to show that the non-zero eigenvalues of $\mathrm{mm}^{T}$ and $m^{T} m$ are equal and in exact one-to-one correspondence. The presence of non-zero $F$-term scalar masses does not spoil this correspondence when $V=0$ : they simply give mass to zero eigenvalues of $m^{T} m$.

${ }^{4}$ This is intuitively clear: (scalar) field excitations along the flat direction are massless while excitations transverse to the flat direction are massive. In the presence of a FI term, however, the flat direction can be reduced to a single point, with all scalar field excitations massive.
}

teractions of the light particles can be described by an effective superpotential which contains only the light degrees of freedom. (The moduli fields associated with the flat direction in question are absent from this superpotential.) In addition to the trilinear couplings of the original superpotential, effective renormalizable interactions for the light fields may also be generated via

$$
W \sim \Psi_{i} \Psi_{j} \Psi_{k}\left(\Pi_{l \in P} \Phi_{l}\right)
$$

In principle, effective nonrenormalizable terms at each order will also be generated from higher-order nonrenormalizable terms in the superpotential in this way. However, there are many other sources for effective nonrenormalizable terms (such as via the decoupling of the heavy fields [25]) which are competitive in strength. A complete classification of the effective nonrenormalizable terms is beyond the scope of this paper.

The method for determining the effective mass terms and trilinear interactions for each flat direction is similar to the strategy for determining the $W_{B}$ terms in the superpotential when testing for $F$-flatness. First, we construct all the bilinear and trilinear terms which are gauge invariant under the unbroken gauge group of the model after the vacuum restabilization [for the $P_{2} P_{3}$ and $P_{1} P_{2} P_{3}$ directions, this includes $U(1)_{Y}$ and $U(1)^{\prime}$ as well as the non-Abelian gauge groups]. We then treat each term as a composite field, and construct all possible terms that are gauge invariant under all of the $U(1)$ 's in the theory [including the anomalous $U(1)$ ] which involve the fields in the flat direction and are linear in the composite field. The next step is to calculate explicitly whether each gauge invariant term is present in the superpotential or forbidden by string selection rules. In practice, the requirements of gauge invariance give the order to which the superpotential must be calculated to determine the full effective renormalizable superpotential.

Once the mass terms are determined, it is straightforward to determine the complete mass spectrum of the model. The trilinear interaction terms are then written in terms of the mass eigenstates, so that the decoupling theorem can be applied to the terms involving the superheavy fields.

In free fermionic constructions the elementary trilinear superpotential terms have coupling strengths $\mathcal{O}(g)$ : the typical value is given by $\sqrt{2} g_{\text {string }}=g$, where again $g$ is the gauge coupling. However, the introduction of the "Ising worldsheet fields" in more involved constructions (e.g., in [11]) allows also for Yukawa couplings $g / \sqrt{2}$ and $g / 2$ [16]. In general, the coefficients $\left(\equiv \alpha_{K+3} / M_{P l}^{K}\right) \quad(K>0)$ of the nonrenormalizable superpotential terms of order $K+3$ are given by the relation

$$
\begin{aligned}
\frac{\alpha_{K+3}}{M_{P l}^{K}} & =g_{\text {string }}\left(\frac{g_{\text {string }}}{2 \pi}\right)^{K}\left(\sqrt{2 \alpha^{\prime}}\right)^{K} C_{K} I_{K} \\
& =g_{\text {string }}\left(\sqrt{\frac{8}{\pi}}\right)^{K} \frac{C_{K} I_{K}}{M_{P l}^{K}},
\end{aligned}
$$


where $\alpha^{\prime}=16 \pi^{2} /\left(g_{\text {string }}^{2} M_{P l}\right)$ is the inverse string tension, $C_{K}$ is a coefficient of $\mathcal{O}(1)$ which includes different renormalization factors in the operator product expansion (OPE) of the string vertex operators (including the target space gauge group Clebsch-Gordan coefficients), and $I_{K}$ is a world-sheet integral. The values of $I_{1}$ and $I_{2}$ have been computed numerically by several authors [16] with the typical values that are in the range $I_{1} \sim 70, I_{2} \sim 400$. (See [37] where special attention is paid to restoring the correct factors and units.)

The coefficients of the effective trilinear terms are then given by $\sim\left(\alpha_{K+3} / M_{P l}^{K}\right)\left|\varphi_{i}\right|^{K}$. Using $x$ as the typical scale of the VEV's, we find that the terms from the fourth order have effective Yukawa coupling strengths $\sim 0.8 C_{1}$, while the fifth order terms have coupling strengths $\sim 0.1 C_{2}$. (We took $\sqrt{2} g_{\text {string }}=g \sim 0.8$, since this is the value typically obtained for the model discussed.) Therefore, compared to the typical elementary trilinear term $\sqrt{2} g_{\text {string }}=g \sim 0.8$ the fourth order terms are competitive in strength to the trilinear terms of the original superpotential, while the higher order contributions are suppressed. Of course, the precise values for each term will depend on the particular fields involved. In particular, the coupling strengths can depend on the free VEV's of the flat direction, and hence are parameters that can be varied in the analysis of the model.

The structure of the effective trilinear couplings strongly depends on the flat direction under consideration. We have determined the mass spectrum and the effective trilinear superpotential terms (in the observable sector) for the directions classified in Table II [33]. In the following, we analyze the details of the two flat directions, $P_{1}^{\prime} P_{2}^{\prime} P_{3}^{\prime}$ and $\left.P_{2} P_{3}\right|_{F}$, as representative examples. These directions encompass general features of the whole class of flat directions (Table II) and demonstrate the nature of the massless spectrum and its phenomenological implications. The first flat direction is "minimal" in the sense that there are a minimal number of surviving trilinear couplings of the massless observable sector. The second one has a richer structure of such couplings, with implications for, e.g., the fermion texture, and baryon and lepton violating processes.

\section{III. $P_{1}^{\prime} P_{2}^{\prime} P_{3}^{\prime}$ FLAT DIRECTION}

\section{A. Effective superpotential}

This flat direction involves the set of fields $P_{1}^{\prime} P_{2}^{\prime} P_{3}^{\prime}$ $=\left\{\varphi_{2}, \varphi_{5}, \varphi_{10}, \varphi_{13}, \varphi_{27}, \varphi_{29}, \varphi_{30}\right\}$ (see Table II). The VEV's for the fields correspond to the most general case given in Eq. (13), such that they depend on two free parameters [which are constrained to be bounded from above by the value of the FI term as dictated by Eq. (15)].

The effective mass terms are computed for this flat direction using the techniques described in the previous section, with the result ${ }^{5}$

$$
\begin{aligned}
W_{M}= & g h_{f} \bar{h}_{b}\left\langle\varphi_{27}\right\rangle+g h_{g} \bar{h}_{d}\left\langle\varphi_{29}\right\rangle+\frac{\alpha_{4}^{(1)}}{M_{P l}} h_{b} \bar{h}_{b}\left\langle\varphi_{5} \varphi_{10}\right\rangle+\frac{\alpha_{4}^{(2)}}{M_{P l}} h_{b} \bar{h}_{b}\left\langle\varphi_{2} \varphi_{13}\right\rangle+\frac{g}{\sqrt{2}}\left(e_{d}^{c} e_{b}+e_{g}^{c} e_{a}\right)\left\langle\varphi_{30}\right\rangle+\frac{g}{\sqrt{2}}\left(\varphi_{1} \varphi_{15}+\varphi_{4} \varphi_{9}\right) \\
& \times\left\langle\varphi_{10}\right\rangle+\frac{g}{\sqrt{2}}\left(\varphi_{7} \varphi_{16}+\varphi_{9} \varphi_{12}\right)\left\langle\varphi_{2}\right\rangle+\frac{g}{\sqrt{2}}\left(\varphi_{6} \varphi_{26}+\varphi_{8} \varphi_{23}+\varphi_{14} \varphi_{17}\right)\left\langle\varphi_{29}\right\rangle+\frac{\alpha_{4}^{(3)}}{M_{P l}} \varphi_{21} \varphi_{25}\left\langle\varphi_{27} \varphi_{29}\right\rangle+\frac{g}{\sqrt{2}}\left(\bar{F}_{1} F_{1}+\bar{F}_{2} F_{2}\right) \\
& \times\left\langle\varphi_{30}\right\rangle+\frac{g}{\sqrt{2}} S_{3} S_{5}\left\langle\varphi_{5}\right\rangle+\frac{g}{\sqrt{2}} S_{1} S_{5}\left\langle\varphi_{13}\right\rangle .
\end{aligned}
$$

The coefficients of the elementary trilinear terms, equal to $g$ or $g / \sqrt{2}$, are displayed explicitly. It is straightforward to determine the mass eigenstates, and we list the massive and massless states in Table III. (The mass spectrum of fields with non-zero VEV's in the flat directions were discussed in Sec. II A and are not explicitly displayed in the tables.)

We then determine the effective trilinear couplings involving the observable sector fields. In addition, we inspect the effective trilinear self-couplings of the non-Abelian singlets and the effective trilinear couplings of non-Abelian singlets to the hidden sector fields which could affect the renormalization group equation (RGE) for the couplings in the observable sector. The result is the following:

\footnotetext{
${ }^{5}$ The effective couplings in this and the subsequent section involve third and fourth order couplings that are modified from those quoted in [11]. This modification is due to two effects: (i) the correctly implemented picture changing procedure in the calculation of couplings introduces a number of additional couplings at the fourth order, and (ii) the implementation of the tests calculating contributions to the correlation functions from the real left-moving world-sheet fermions excludes a number of couplings involving some of the non-Abelian hidden sector fields. The full superpotential up to the fifth order will be presented elsewhere.
} 


$$
\begin{aligned}
W_{3}= & g Q_{c} u_{c}^{c} \bar{h}_{c}+g Q_{c} d_{b}^{c} h_{c}+\frac{\alpha_{4}^{(4)}}{M_{P l}} Q_{c} d_{d}^{c} h_{a}\left\langle\varphi_{29}\right\rangle+\frac{g}{\sqrt{2}} e_{a}^{c} h_{a} h_{c}+\frac{g}{\sqrt{2}} e_{f}^{c} h_{d} h_{c}+\frac{\alpha_{5}^{(1)}}{M_{P l}^{2}} e_{h}^{c} h_{e} h_{a}\left\langle\varphi_{5} \varphi_{27}\right\rangle+\frac{\alpha_{5}^{(2)}}{M_{P l}^{2}} e_{e}^{c} h_{e} h_{a}\left\langle\varphi_{13} \varphi_{27}\right\rangle \\
& +g h_{f} \bar{h}_{c} \varphi_{22}+g h_{b} \bar{h}_{c} \varphi_{20}+\frac{\alpha_{4}^{(5)}}{M_{P l}} S_{2} S_{6} \varphi_{20}\left\langle\varphi_{27}\right\rangle .
\end{aligned}
$$

The result is expressed in terms of the mass eigenstates and the VEV's, and the decoupling theorem is applied to the terms involving the heavy fields:

$$
\begin{aligned}
W_{3}= & g Q_{c} u_{c}^{c} \bar{h}_{c}+g Q_{c} d_{b}^{c} h_{c}+\frac{g}{\sqrt{2}} e_{a}^{c} h_{a} h_{c}+\frac{g}{\sqrt{2}} e_{f}^{c} h_{d} h_{c}+\frac{\sqrt{2} \alpha_{5}^{(1)} x^{2}}{M_{P l}^{2}} \lambda_{2} e_{h}^{c} h_{e} h_{a}+\frac{\sqrt{2} \alpha_{5}^{(2)} x^{2}}{M_{P l}^{2}} \sqrt{\lambda_{1}^{2}-\lambda_{2}^{2}} e_{e}^{c} h_{e} h_{a}+g \bar{h}_{c} h_{b}^{\prime} \varphi_{20}^{\prime} \\
& +\frac{\alpha_{4}^{(4)}}{M_{P l}} \sqrt{1-\lambda_{1}^{2}} Q_{c} d_{d}^{c} h_{a}+\frac{\sqrt{2} \alpha_{4}^{(5)} x}{M_{P l}} \frac{1}{\sqrt{1+r^{2}}}\left(\varphi_{20}^{\prime}+r \varphi_{22}^{\prime}\right) S_{2} S_{6},
\end{aligned}
$$

in which

$$
\begin{gathered}
\lambda_{2} \equiv \frac{\left|\psi_{2}\right|}{x} \leqslant \lambda_{1} \equiv \frac{\left|\psi_{1}\right|}{x} \leqslant 1, \\
\varphi_{20}^{\prime}=\left[1 /\left(\sqrt{1+r^{2}}\right)\right]\left(\varphi_{20}-r \varphi_{22}\right)
\end{gathered}
$$

and

$$
\varphi_{22}^{\prime}=\left[1 /\left(\sqrt{1+r^{2}}\right)\right]\left(r \varphi_{20}+\varphi_{22}\right),
$$

with $r \equiv\left[\alpha_{4}^{(1)} \lambda_{2}^{2}+\alpha_{4}^{(2)}\left(\lambda_{1}^{2}-\lambda_{2}^{2}\right)\right] x /\left(\sqrt{2} g M_{P l}\right)$. Here $h_{b}^{\prime}$ is defined in Table III(b). In the numerical analysis, $\lambda_{1}, \lambda_{2}$ are parameters that can be varied.

\section{B. Implications}

The effective superpotential has a number of interesting implications.

\section{Massless states}

There are a large number of states that remain massless, as indicated in Table III(b). These states include both the usual MSSM states and related exotic [non-chiral under $\left.S U(2)_{L}\right]$ states, such as a fourth $\left[S U(2)_{L}\right.$ singlet] down-type quark, extra fields with the same quantum numbers as the lepton singlet superfields, and extra Higgs doublets. There are other massless states with exotic quantum numbers (including fractional electric charge) and states which are nonAbelian representations under both the hidden and observable sector gauge groups and thus directly mix the two sectors. The scalar components of these superfields may acquire masses via soft supersymmetry breaking. However, within our set of assumptions there is no mechanism to give many of the fermions significant masses. ${ }^{6}$ As discussed ear-

\footnotetext{
${ }^{6}$ One possible mechanism is to invoke a non-minimal Kähler potential. Another one, which is not possible for this particular flat direction, is to utilize an intermediate scale, as discussed in the next section.
}

lier, there are additional massless states (moduli) associated with the fields which appear in the flat direction but which are not fixed. These are not listed in Table III(b).

\section{U(1)' charges of light fields}

To invoke an intermediate scale $U(1)^{\prime}$ symmetry breaking scenario (as discussed in [33]), which can lead to a mechanism to give significant masses to the additional light fields via higher-dimension operators, it is necessary to have at least one pair of $Y=0$ singlets which remain massless after vacuum restabilization which have $U(1)^{\prime}$ charges opposite in sign (to allow for the breaking to occur along a $D$-flat direction). An inspection of Table I(c) indicates that the singlet field $\varphi_{25}$ is required for this scenario; however, this field acquires a string-scale mass for this direction, and decouples from the theory. We conclude that in this case, an intermediate scale scenario is not possible, and hence the breaking of the $U(1)^{\prime}$ is necessarily at the electroweak scale. [We do not consider more complicated scenarios in which the $U(1)^{\prime}$ could be broken along with some of the hidden non-Abelian groups.]

As discussed in [28,30,31], several scenarios exist which can lead to the possibility of a realistic $Z-Z^{\prime}$ hierarchy. The scenario in which only the two MSSM Higgs fields $h_{c}, \bar{h}_{c}$ acquire VEV's breaks both $U(1)_{Y}$ and $U(1)^{\prime}$ [because the $U(1)$ charges of these fields are not equal and opposite], but leads to a $Z^{\prime}$ which is $\mathcal{O}\left(M_{Z}\right)$, which is already excluded. The scenario in which the symmetry breaking is driven by a large trilinear coupling (described in [30]) is also not feasible because the $U(1)^{\prime}$ charges of the relevant Higgs fields (which have $Y= \pm 1 / 2$ ) are opposite in sign, and thus do not allow for a small mixing angle. Therefore, the only remaining possibility is to have a scenario in which the symmetry breaking is characterized by a large $[\mathcal{O}(\mathrm{TeV})] \mathrm{SM}$ singlet $\operatorname{VEV}\left(\left\langle\varphi_{20}^{\prime}\right\rangle\right)$, with the $S U(2)_{L} \times U(1)_{Y}$ breaking at a lower scale due to accidental cancellations.

In addition, the $U(1)^{\prime}$ charges of the observable sector 
TABLE III. (a) List of massive states for the $P_{1}^{\prime} P_{2}^{\prime} P_{3}^{\prime}$ flat direction as can be read off from the effective bilinear superpotential terms (19). Here $N_{1} \equiv 2 g^{2} x^{2}+\left[\alpha_{4}^{(1)}\left|\psi_{2}\right|^{2}+\alpha_{4}^{(2)}\left(\left|\psi_{1}\right|^{2}-\left|\psi_{2}\right|^{2}\right)\right]^{2} / M_{P l}^{2}$ and $N_{2} \equiv 2\left(\alpha_{4}^{(3)} / M_{p l}\right)^{2} x^{2}\left(x^{2}-\left|\psi_{1}\right|^{2}\right)$ and the definition of VEV parameters $x, \psi_{1}$ and $\psi_{2}$ is given in Eqs. (13). The fields with nonzero VEV's in the flat directions $\left(\varphi_{2}, \varphi_{5}, \varphi_{10}, \varphi_{13}, \varphi_{27}, \varphi_{29}, \varphi_{30}\right)$ are discussed in the text (at the end of Sec. ibid. B) and are not given in this table. (Five complex fields contribute to the Higgs mechanism, completing five massive vector supermultiplets, associated with the spontaneous symmetry breaking of five $U(1)$ factors [including anomalous $U(1)$ ] and two complex fields remain massless (and act as moduli associated with the two free parameters of VEV's).) (b) List of massless states (excluding the two moduli) for the $P_{1}^{\prime} P_{2}^{\prime} P_{3}^{\prime}$ flat direction. These are fields without an effective bilinear term in the superpotential (19).

Massive fields

$\bar{h}_{b}, h_{f}^{\prime}=\frac{1}{\sqrt{N_{1}}}\left[\sqrt{2} g x h_{f}+\frac{\left(\alpha_{4}^{(1)}\left|\psi_{2}\right|^{2}+\alpha_{4}^{(2)}\left(\left|\psi_{1}\right|^{2}-\left|\psi_{2}\right|^{2}\right)\right.}{M_{P l}} h_{b}\right]$

$h_{g}, \bar{h}_{d}$

$e_{d}^{c}, e_{g}^{c}, e_{a}, e_{b}$

$\varphi_{9}, \varphi_{4}^{\prime}=\frac{1}{\left|\psi_{1}\right|}\left(\left|\psi_{2}\right| \varphi_{4}+\sqrt{\left|\psi_{1}\right|^{2}-\left|\psi_{2}\right|^{2}} \varphi_{12}\right)$

$\varphi_{1}, \varphi_{15}$

$\varphi_{7}, \varphi_{16}$

$\varphi_{6}, \varphi_{8}, \varphi_{14}, \varphi_{17}, \varphi_{23}, \varphi_{26}$

$\varphi_{21}, \varphi_{25}$

$F_{1}, F_{2}, \bar{F}_{1}, \bar{F}_{2}$

$S_{5}, S_{3}^{\prime}=\frac{1}{\left|\psi_{1}\right|}\left(\left|\psi_{2}\right| S_{3}+\sqrt{\left|\psi_{1}\right|^{2}-\left|\psi_{2}\right|^{2}} S_{1}\right)$ (a)

Mass

$$
\begin{gathered}
g \sqrt{x^{2}-\left|\psi_{1}\right|^{2}} \\
\frac{g}{\sqrt{2}}\left|\psi_{1}\right| \\
\frac{g}{\sqrt{2}}\left|\psi_{1}\right| \\
\frac{g}{\sqrt{2}}\left|\psi_{2}\right| \\
\frac{g}{\sqrt{2}} \sqrt{\left|\psi_{1}\right|^{2}-\left|\psi_{2}\right|^{2}} \\
\frac{g}{\sqrt{2}} \sqrt{x^{2}-\left|\psi_{1}\right|^{2}} \\
\frac{\sqrt{N_{2}}}{\sqrt{2}}\left|\psi_{1}\right| \\
\frac{g}{\sqrt{2}}\left|\psi_{1}\right|
\end{gathered}
$$

(b)

Massless Fields

$$
\begin{aligned}
& Q_{a}, Q_{b}, Q_{c} \\
& u_{a}^{c}, u_{b}^{c}, u_{c}^{c} \\
& d_{a}^{c}, d_{b}^{c}, d_{c}^{c}, d_{d}^{c}, \mathcal{D}_{a} \\
& \bar{h}_{a}, \bar{h}_{c} \\
& h_{a}, h_{c}, h_{d}, h_{e} \\
& h_{b}^{\prime}=\frac{1}{\sqrt{N_{1}}}\left[-\frac{\alpha_{4}^{(1)}\left|\psi_{2}\right|^{2}+\alpha_{4}^{(2)}\left(\left|\psi_{1}\right|^{2}-\left|\psi_{2}\right|^{2}\right)}{M_{P l}} h_{f}+\sqrt{2} g x h_{b}\right] \\
& e_{a}^{c}, e_{b}^{c}, e_{c}^{c}, e_{e}^{c}, e_{f}^{c}, e_{h}^{c}, e_{i}^{c} \\
& e_{c}, e_{d}, e_{e}, e_{f} \\
& \varphi_{3}, \varphi_{11}, \varphi_{18}, \varphi_{19}, \varphi_{20}, \varphi_{22}, \varphi_{24}, \varphi_{28} \\
& \varphi_{12}^{\prime}=\frac{1}{\left|\psi_{1}\right|}\left(-\sqrt{\left|\psi_{1}\right|^{2}-\left|\psi_{2}\right|^{2}} \varphi_{12}+\left|\psi_{2}\right| \varphi_{4}\right) \\
& \bar{F}_{3}, \bar{F}_{4}, \bar{F}_{5}, \bar{F}_{6}, \bar{F}_{7}, \bar{F}_{8}, \bar{F}_{9}, \bar{F}_{10} \\
& H_{1}, H_{2}, H_{3}, H_{4}, H_{5}, H_{6}, H_{7}, H_{8} \\
& \bar{E}_{1}, E_{1}, E_{2}, E_{3}, E_{4}, E_{5} \\
& S_{2}, S_{4}, S_{6}, S_{7}, S_{8} \\
& \begin{array}{c}
S_{1}^{\prime}=\frac{1}{\left|\psi_{1}\right|}\left(-\sqrt{\left|\psi_{1}\right|^{2}-\left|\psi_{2}\right|^{2}} S_{3}+\left|\psi_{2}\right| S_{1}\right) \\
T_{1}, T_{2}, T_{3}
\end{array}
\end{aligned}
$$



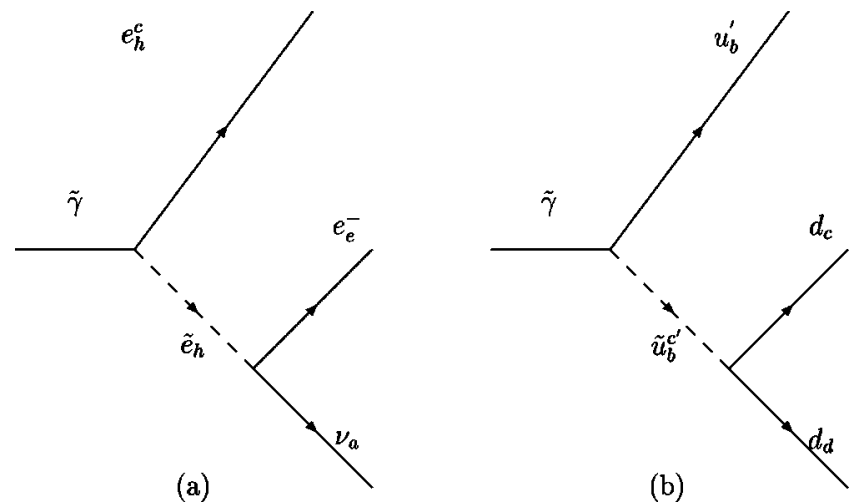

FIG. 1. Diagram for gaugino decay into (a) three leptons $(\Delta L$ =1), which occurs for the $P_{1}^{\prime} P_{2}^{\prime} P_{3}^{\prime}$ direction (there are similar diagrams for decay into $t d_{d}^{c} e_{a}^{-}$or $b d_{d}^{c} \nu_{a}$ ), and (b) three quarks $(\Delta B=1)$, which occurs for the $\left.P_{2} P_{3}\right|_{F}$ direction. $u_{b}^{\prime}, d_{c}$ and $d_{d}$ are $S U(2)$ singlets (i.e., the conjugates of $u_{b}^{c^{\prime}}, d_{c}^{c}$ and $d_{d}^{c}$ ).

fields indicate that the $Z^{\prime}$ couplings are family nonuniversal. In the quark sector, the largest couplings are to the third family, with smaller (equal) couplings to the first two. There is a large coupling to the exotic $\mathcal{D}_{a}$. In the lepton sector, where the family assignments are less clear, there are unequal couplings to all families. Family mixing between the quarks or between the leptons would lead to flavor changing neutral current (FCNC) effects in the $Z^{\prime}$ and (through $Z-Z^{\prime}$ mixing) $Z$ couplings, while mixing with exotics would induce FCNC for both the $Z$ and $Z^{\prime}$ directly. (Of course, the present model does not have a satisfactory way to introduce such family mixings.)

\section{L violation}

The doublets $h_{a}, h_{b}^{\prime}, h_{c}, h_{d}$, and $h_{e}$ can, in principle (i.e., before examining their superpotential couplings), be identified as either Higgs or lepton doublets. $h_{c}$, as well as $\bar{h}_{c}$, should clearly be identified as Higgs doublets from their couplings to ordinary quarks. We will also identify $h_{b}^{\prime}$ as a Higgs doublet, so that the $\bar{h}_{c} h_{b}^{\prime} \varphi_{20}^{\prime}$ term conserves lepton number. The remaining $h_{a}, h_{d}$ and $h_{e}$ are candidates for lepton doublets. In particular, the couplings of $h_{a}$ and $h_{d}$ to the Higgs doublet $h_{c}$ indicate that these are the doublets corresponding to the $\mu$ and $\tau$ leptons. There is no difficulty with $h_{d}$. However, the leptoquark coupling $Q_{c} d_{d}^{c} h_{a}$ [in which $Q_{c}=(t, b)^{T}$ and $d_{d}^{c}$ can be either $d^{c}, s^{c}$, or the exotic quark $\left.D^{c}\right]$ as well as the $e_{h}^{c} h_{e} h_{a}$ and $e_{e}^{c} h_{e} h_{a}$ terms would then violate lepton number by one unit, and similarly there is no conserved $R$ parity in this model. (The strength of the $L$-violating coupling $Q_{c} d_{d}^{c} h_{a}$, coming from the 4 th order, could be reduced by choosing the free parameter $\lambda_{1}$ close to 1 , while allowing the relevant massive fields to still have string scale masses.)

One consequence of these couplings is that there is no stable LSP in this model. For example, a neutral gaugino could decay into the fermion $e_{h}^{c}$ and its (virtual) scalar conjugate $\tilde{e}_{h}$, followed by $\tilde{e}_{h} \rightarrow e_{e}^{-} \nu_{a}$ or $e_{a}^{-} \nu_{e}$, where we use

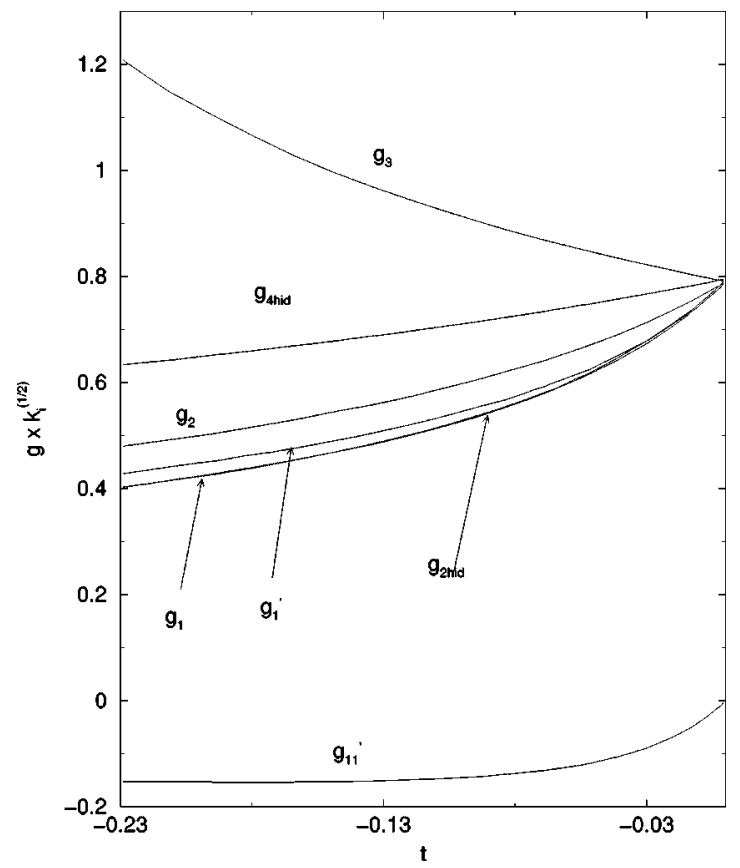

FIG. 2. Running of the gauge couplings $\times \sqrt{k}$ for the $P_{1}^{\prime} P_{2}^{\prime} P_{3}^{\prime}$ flat direction, with $t=1 / 16 \pi^{2} \ln \left(\mu / M_{\text {string }}\right)$, with $M_{\text {string }}$ $=5 \times 10^{17} \mathrm{GeV}, g_{\mid M_{\text {string }}}=0.80$. The couplings include the factor $\sqrt{k}$, where $k$ corresponds to the Kač-Moody level (see the caption of Table $\mathrm{V}$ for the values of $k$ ).

the notation $h_{a, e}=\left(\nu, e^{-}\right)_{a, e}^{T}$ [see Fig. 1(a)]. In principle, Majorana neutrino mass terms can also be generated at one loop from the $e_{h, e}^{c} h_{e} h_{a}$ or $Q_{c} d_{d}^{c} h_{a}$ coupling. However, such terms are absent in this model because a nonzero VEV for $h_{e}$ or $h_{a}$ is required. ${ }^{7}$

\section{Effective Yukawa couplings}

The superpotential not only does not have an elementary $\mu$ term, but also does not have the usual effective $\mu$ term [28-30] of the form $\bar{h}_{c} h_{c} \varphi$ for any SM singlet $\varphi$. Gauge invariance and string selection rules forbid the presence of this effective $\mu$ term for all of the flat directions considered for this model. There is, however, a non-canonical $\mu$ term $\bar{h}_{c} h_{b}^{\prime} \varphi_{20}^{\prime}$, which couples to only one of the the ordinary Higgs doublets $\left(h_{c}\right.$ and $\left.\bar{h}_{c}\right)$. In a subsequent paper [27] we shall analyze the running of the Yukawa couplings and an acceptable gauge symmetry breaking pattern that can be obtained even without the soft breaking " $B \mu$ " found in the MSSM. However, the lack of a $\mu$ term of the form $\bar{h}_{a} h_{c} \varphi$ leads to an unwanted massless chargino and neutralino, while the absence of this or of a canonical $\mu$ term of the type $\bar{h}_{c} h_{c} \varphi$ leads to a second almost massless neutralino and an unwanted approximate global $U(1)$ symmetry.

\footnotetext{
${ }^{7}$ For a discussion of neutrino masses in models with $R$ parity violation, see [38].
} 
TABLE IV. (a) List of massive states for the $\left.P_{2} P_{3}\right|_{F}$ flat direction, with $N_{1 / 2} \equiv g^{2} x^{2} / 4$ $+\left(\alpha_{5}^{(1 / 2)} x^{3} / 2 M_{P l}^{2}\right)^{2}$. Except for $\varphi_{9}$ the effective bilinear terms can be read off Eq. (24). The fields with nonzero VEV's in the flat directions $\left(\varphi_{2}, \varphi_{4}, \varphi_{10}, \varphi_{12}, \varphi_{27}, \varphi_{30}\right)$ are discussed in the text (at end of Sec. ibid. B) and are not given in this table. (Five complex fields contribute to the Higgs mechanism, completing five massive vector supermultiplets, associated with the spontaneous symmetry breaking of five $U(1)$ factors [including anomalous $U(1)$ ] and one complex field gets its mass due to the superpotential terms which impose $F$ - flatness constraints on VEV's, i.e., $(g x / 2) \varphi_{9}\left(\delta \varphi_{2}+\delta \varphi_{12}-\delta \varphi_{4}+\delta \varphi_{10}\right)$. Note that $\varphi_{9}$ acquires mass due to the same coupling in the superpotential.) $9 \mathrm{~b}$ ) List of massless states [without effective bilinear terms in Eq. (24)] for the $\left.P_{2} P_{3}\right|_{F}$ flat direction.

(a)

Massive fields

Mass

$\varphi_{9}$

$g x$

$\bar{h}_{b}, h_{f}$

$\sqrt{2} g x$

$e_{d}^{c}, e_{g}^{c}, e_{a}, e_{b}$

$\frac{g}{\sqrt{2}} x$

$\varphi_{8}, \varphi_{3^{\prime}}=\left(\varphi_{3}+\varphi_{11}\right) / \sqrt{2}$

$\frac{g}{\sqrt{2}} x$

$\varphi_{15}, \varphi_{1^{\prime}}=\left(-\frac{g x}{2} \varphi_{1}+\frac{\alpha_{5}^{(1)}}{M_{P l}^{2}} \frac{x^{3}}{2} \varphi_{26}\right) / \sqrt{N_{1}}$

$\sqrt{N_{1}}$

$\varphi_{7}, \varphi_{16^{\prime}}=\left(\frac{g x}{2} \varphi_{16}-\frac{\alpha_{5}^{(2)}}{M_{P l}^{2}} \frac{x^{3}}{2} \varphi_{17}\right) / \sqrt{N_{2}}$

$\sqrt{N_{2}}$

$F_{1}, F_{2}, \bar{F}_{1}, \bar{F}_{2}$

$\frac{g}{\sqrt{2}} x$

(b)

$$
\begin{gathered}
Q_{a}, Q_{b}, Q_{c} \\
u_{a}^{c}, u_{b}^{c}, u_{c}^{c} \\
d_{a}^{c}, d_{b}^{c}, d_{c}^{c}, d_{d}^{c}, \mathcal{D}_{a} \\
\bar{h}_{a}, \bar{h}_{c}, \bar{h}_{d} \\
h_{a}, h_{b}, h_{c}, h_{d}, h_{e}, h_{g} \\
e_{a}^{c}, e_{b}^{c}, e_{c}^{c}, e_{e}^{c}, e_{f}^{c}, e_{h}^{c}, e_{i}^{c} \\
e_{c}, e_{d}, e_{e}, e_{f} \\
\varphi_{5}, \varphi_{6}, \varphi_{13}, \varphi_{14}, \varphi_{18}, \varphi_{19}, \varphi_{20}, \varphi_{21}, \varphi_{22}, \varphi_{23}, \varphi_{24}, \varphi_{25}, \varphi_{28}, \varphi_{29} \\
\varphi_{11^{\prime}}=\left(\varphi_{3}-\varphi_{11}\right) / \sqrt{2}, \\
\varphi_{17^{\prime}}=\left(\begin{array}{c}
g x \\
\left.\frac{g x}{2} \varphi_{17}+\frac{\alpha_{5}^{(2)}}{M_{P l}^{2}} \frac{x^{3}}{2} \varphi_{16}\right) / \sqrt{N_{2}} \\
\varphi_{26^{\prime}}=\left(\begin{array}{c}
g x \\
\left.-\frac{\alpha_{5}^{(1)}}{2} \varphi_{26}-\frac{x^{3}}{M_{P l}^{2}} \varphi_{1}\right) / \sqrt{N_{1}} \\
D_{1}, D_{2}, D_{3}, D_{4}
\end{array}\right. \\
\bar{F}_{3}, \bar{F}_{4}, \bar{F}_{5}, \bar{F}_{6}, \bar{F}_{7}, \bar{F}_{8}, \bar{F}_{9}, \bar{F}_{10} \\
H_{1}, H_{2}, H_{3}, H_{4}, H_{5}, H_{6}, H_{7}, H_{8} \\
\bar{E}_{1}, E_{1}, E_{2}, E_{3}, E_{4}, E_{5} \\
S_{1}, S_{2}, S_{3}, S_{4}, S_{5}, S_{6}, S_{7}, S_{8} \\
T_{1}, T_{2}, T_{3}
\end{array}\right.
\end{gathered}
$$

Massless fields 
In addition, the superpotential for this direction has the feature that only the third quark family has large Yukawa couplings, which is a desirable feature. However, the Yukawa couplings also indicate $t-b$ and $\tau$ - $\mu$ Yukawa unification with the equal string scale Yukawa couplings $g$ and $g / \sqrt{2}$, respectively. ${ }^{8}$ Unfortunately, the ratio of $b$ and $\tau$ string scale Yukawa couplings $1 / \sqrt{2}$ is probably not consistent with the observed $m_{b} / m_{\tau}$ ratio [39], and the $\tau$ - $\mu$ unification is clearly in disagreement with experiment since it would lead to approximately equal $\tau$ and $\mu$ masses. The $t-b$ unification may be acceptable, but only for a sufficiently large $\tan \beta$, where $\tan \beta$ is the ratio of VEV's of the neutral components of the $\bar{h}_{c}$ and $h_{c}$ scalars [39]. [The MSSM mass relations may be modified because the sum of the squares of the VEVs of the doublets related to the fermion masses $\left(h_{c}, \bar{h}_{c}\right)$ may be reduced due to the presence of additional Higgs doublets.] The superpotential also does not have Yukawa textures in the quark sector, so that the first and second quark families (as well as the first lepton) remain massless.

\section{Running of gauge couplings}

From the massless particle content listed in Table III(b), the $\beta$ functions for the running of the gauge couplings can easily be computed. As the number of additional $S U(3)_{C}$ exotic fields is minimal (one vectorlike pair), the running of $g_{3}$ is closer to that of the MSSM than the other gauge couplings. Therefore, our strategy is to take the value of $g_{3}$ at the electroweak scale as an input (we choose $\alpha_{s}=0.12$ at $M_{Z}$ ), and run the couplings to the string scale to determine the value of $g=0.80$. (For our purpose, it is adequate to consider RGE's at the one-loop level, ignoring SUSY threshold effects.) The other gauge couplings are then run back to the electroweak scale with this value of $g$ as an input, taking into account the Kač-Moody level for the $U(1)$ gauge factors $\left(k_{Y}=11 / 3, k_{Y}^{\prime} \simeq 16.67\right)$ and for the hidden sector nonAbelian groups $(k=2)$.
In this case, it would appear from the massless particle content that the running of the gauge couplings for $S U(2)_{L}$ and $U(1)_{Y}$ are very different from the MSSM case. However, we find that the low energy values yield a prediction for $\sin ^{2} \theta_{W} \sim 0.16$. While this is lower than the experimental value $(\sim 0.23)$, the disagreement is less than might have been expected given the large amount of exotic matter and the value $k_{Y}=11 / 3$ (to be compared with the MSSM value $5 / 3)$. Similarly, for the $S U(2)$ gauge coupling, we find $g_{2}$ $=0.48$ surprisingly close to the experimental value $\sim 0.65$. The variation of the gauge couplings with the scale is presented in Fig. 2, and the $\beta$ functions are listed in Table V, below.

In addition, the hidden sector gauge groups are not asymptotically free, and hence there is no possibility for gaugino condensation or other strong coupling dynamics to break supersymmetry in the hidden sector.

\section{IV. $\left.P_{2} P_{3}\right|_{F}$ FLAT DIRECTION}

The fields involved in this flat direction are $\left.P_{2} P_{3}\right|_{F}$ $=\left\{\varphi_{2}, \varphi_{4}, \varphi_{10}, \varphi_{12}, \varphi_{27}, \varphi_{30}\right\}$ (see Table II). The VEV's of these fields are completely fixed due to the $F$-flatness constraints:

$$
\begin{gathered}
\left|\left\langle\varphi_{27}\right\rangle\right|^{2}=2 x^{2}, \\
\left|\left\langle\varphi_{30}\right\rangle\right|^{2}=2\left|\left\langle\varphi_{2}\right\rangle\right|^{2}=2\left|\left\langle\varphi_{4}\right\rangle\right|^{2}=2\left|\left\langle\varphi_{10}\right\rangle\right|^{2}=2\left|\left\langle\varphi_{12}\right\rangle\right|^{2}=x^{2},
\end{gathered}
$$

where $x=0.013 M_{P l}$, and $\varphi_{10}$ and $\varphi_{4}$ have opposite signs of their VEV's. (Recall, that without loss of generality, we take all the VEV's real and, except for $\varphi_{10}$, positive.)

The effective mass terms for this direction are

TABLE V. Effective beta-functions are quoted for the two representative flat directions. The effective beta-function is defined as $\beta_{i} \equiv \beta_{i}^{0} / k_{i}$, where $\beta_{i}^{0}$ and $k_{i}$ are the beta-function and the Kač-Moody level for a particular gauge group factor, respectively. The subscripts 1, 2, 3, 1', 2hid, 4hid refer to $U(1)_{Y}, S U(2)_{L}, S U(3)_{C}, U(1)^{\prime}, S U(2)_{2}, S U(4)_{2}$ gauge group factors and $11^{\prime}$ refers to the $U(1)_{Y}$ and $U(1)^{\prime}$ kinetic mixing. The Kač-Moody levels are $k_{1}=11 / 3, k_{2}=k_{3}=1, k_{1}, \simeq 16.67$, and $k_{2 h i d}=k_{4 h i d}$ $=2$.

\begin{tabular}{lccccccc}
\hline \hline Effective $\beta$ & $\beta_{1}$ & $\beta_{2}$ & $\beta_{3}$ & $\beta_{1^{\prime}}$ & $\beta_{11^{\prime}}$ & $\beta_{2 h i d}$ & $\beta_{4 h i d}$ \\
\hline$P_{1}^{\prime} P_{2}^{\prime} P_{3}^{\prime}$ flat firection & 10.0 & 6.0 & -2.0 & 10.2 & 4.8 & 10.0 & 2.0 \\
$\left.P_{2} P_{3}\right|_{F}$ flat direction & 10.3 & 7.0 & -2.0 & 10.6 & 5.0 & 10.0 & 3.0 \\
\hline \hline
\end{tabular}

\footnotetext{
${ }^{8}$ This type of Yukawa coupling unification is stringy in nature and different from the standard GUT considerations. The obtained hierarchy between the lepton and quark Yukawa couplings is due to the fact that the "canonical" candidates for the lepton doublets (which would have had the same Yukawa couplings as quarks) are massive for the flat directions considered, and thus the lepton Yukawa couplings involve fields that are usually identified with additional copies of the (exotic) Higgs doublets or (exotic) lepton doublets.
} 


$$
\begin{aligned}
W_{M}= & g h_{f} \bar{h}_{b}\left\langle\varphi_{27}\right\rangle+\frac{g}{\sqrt{2}}\left(e_{d}^{c} e_{b}+e_{g}^{c} e_{a}\right)\left\langle\varphi_{30}\right\rangle+\frac{g}{\sqrt{2}} \varphi_{3} \varphi_{8}\left\langle\varphi_{12}\right\rangle+\frac{g}{\sqrt{2}} \varphi_{8} \varphi_{11}\left\langle\varphi_{4}\right\rangle+\frac{g}{\sqrt{2}} \varphi_{7} \varphi_{16}\left\langle\varphi_{2}\right\rangle+\frac{g}{\sqrt{2}} \varphi_{1} \varphi_{15}\left\langle\varphi_{10}\right\rangle \\
& +\frac{\alpha_{5}^{(1)}}{M_{P l}^{2}} \varphi_{15} \varphi_{26}\left\langle\varphi_{2} \varphi_{4} \varphi_{30}\right\rangle+\frac{\alpha_{5}^{(2)}}{M_{P l}^{2}} \varphi_{7} \varphi_{17}\left\langle\varphi_{10} \varphi_{12} \varphi_{30}\right\rangle+\frac{g}{\sqrt{2}} F_{1} \bar{F}_{1}\left\langle\varphi_{30}\right\rangle+\frac{g}{\sqrt{2}} F_{2} \bar{F}_{2}\left\langle\varphi_{30}\right\rangle .
\end{aligned}
$$

As in the previous case, the coefficients of the elementary trilinear terms, equal to $g$ or $g / \sqrt{2}$, are displayed explicitly. The massive and massless states are listed in Table IV(a) and IV(b), respectively. The fields with nonzero VEV's are all massive in this case (the "flat-point" discussed at the end of Sec. IIA) and are not included in Table IV(a).

After decoupling the heavy fields, the effective trilinear couplings for the observable sector states are

$$
\begin{aligned}
W_{3}= & g Q_{c} u_{c}^{c} \bar{h}_{c}+g Q_{c} d_{b}^{c} h_{c}+\frac{\alpha_{5}^{(3)}}{M_{P l}^{2}} Q_{a} d_{d}^{c} h_{g}\left\langle\varphi_{4} \varphi_{30}\right\rangle+\frac{\alpha_{5}^{(4)}}{M_{P l}^{2}} Q_{b} d_{d}^{c} h_{g}\left\langle\varphi_{12} \varphi_{30}\right\rangle+\frac{\alpha_{5}^{(5)}}{M_{P l}^{2}} Q_{b} d_{c}^{c} h_{b}\left\langle\varphi_{12} \varphi_{30}\right\rangle+\frac{\alpha_{5}^{(6)}}{M_{P l}^{2}} Q_{a} d_{c}^{c} h_{b}\left\langle\varphi_{4} \varphi_{30}\right\rangle \\
& +\left(\frac{\alpha_{4}^{(1)}}{M_{P l}} u_{b}^{c}\left\langle\varphi_{4}\right\rangle+\frac{\alpha_{4}^{\left(1^{\prime}\right)}}{M_{P l}} u_{a}^{c}\left\langle\varphi_{12}\right\rangle\right) d_{c}^{c} d_{d}^{c}+\frac{g}{\sqrt{2}} e_{a}^{c} h_{a} h_{c}+\frac{g}{\sqrt{2}} e_{f}^{c} h_{d} h_{c}+\left(\frac{\alpha_{4}^{(2)}}{M_{P l}} e_{e}^{c}\left\langle\varphi_{12}\right\rangle+\frac{\alpha_{4}^{\left(2^{\prime}\right)}}{M_{P l}} e_{h}^{c}\left\langle\varphi_{4}\right\rangle\right) h_{g} h_{b} \\
& +\frac{\alpha_{5}^{(7)}}{M_{P l}^{2}} e_{b}^{c} h_{g} h_{a}\left\langle\varphi_{10} \varphi_{12}\right\rangle+\frac{\alpha_{5}^{(8)}}{M_{P l}^{2}} e_{i}^{c} h_{g} h_{a}\left\langle\varphi_{2} \varphi_{4}\right\rangle+\frac{g}{\sqrt{2}} \bar{h}_{a} h_{c} \varphi_{25}+g \bar{h}_{c} h_{b} \varphi_{20}+g \bar{h}_{d} h_{b} \varphi_{28}+g \bar{h}_{c} h_{g} \varphi_{21}+g \bar{h}_{d} h_{g} \varphi_{29}
\end{aligned}
$$

from which we can redefine two new fields as

$$
\begin{gathered}
u_{b}^{c^{\prime}}=\frac{M_{P l}}{\sqrt{\left(\alpha_{4}^{(1)}\left\langle\varphi_{4}\right\rangle\right)^{2}+\left(\alpha_{4}^{\left(1^{\prime}\right)}\left\langle\varphi_{12}\right\rangle\right)^{2}}}\left(\frac{\alpha_{4}^{(1)}}{M_{P l}}\left\langle\varphi_{4}\right\rangle u_{b}^{c}+\frac{\alpha_{4}^{\left(1^{\prime}\right)}}{M_{P l}}\left\langle\varphi_{12}\right\rangle u_{a}^{c}\right), \\
e_{e}^{c^{\prime}}=\frac{M_{P l}}{\sqrt{\left(\alpha_{4}^{(2)}\left\langle\varphi_{12}\right\rangle\right)^{2}+\left(\alpha_{4}^{\left(2^{\prime}\right)}\left\langle\varphi_{4}\right\rangle\right)^{2}}}\left(\frac{\alpha_{4}^{(2)}}{M_{P l}}\left\langle\varphi_{12}\right\rangle e_{e}^{c}+\frac{\alpha_{4}^{\left(2^{\prime}\right)}}{M_{P l}}\left\langle\varphi_{4}\right\rangle e_{h}^{c}\right) .
\end{gathered}
$$

In addition, there are effective trilinear couplings involving the singlets $\varphi_{i}$ and the hidden sector non-Abelian fields which also have trilinear couplings to the $\varphi_{i}$. Some of these terms play important roles in radiative symmetry breaking scenarios. We quote only these terms and the complete discussion of the trilinear terms in the hidden sector is deferred for further investigation:

$$
\begin{aligned}
W_{3 h i d}= & \frac{\alpha_{4}^{(3)}}{M_{P l}} \varphi_{29} \varphi_{21} \varphi_{25}\left\langle\varphi_{27}\right\rangle+\frac{\alpha_{4}^{\left(3^{\prime}\right)}}{M_{P l}} \varphi_{28} \varphi_{20} \varphi_{25}\left\langle\varphi_{27}\right\rangle+\frac{\alpha_{4}^{(5)}}{M_{P l}} S_{2} S_{7} \varphi_{21}\left\langle\varphi_{27}\right\rangle+\frac{\alpha_{4}^{(6)}}{M_{P l}} S_{2} S_{6} \varphi_{20}\left\langle\varphi_{27}\right\rangle+\frac{\alpha_{6}^{(1)}}{M_{P l}^{3}} S_{4} S_{7} \varphi_{21}\left\langle\varphi_{27} \varphi_{30} \varphi_{12}\right\rangle \\
& +\frac{\alpha_{6}^{(2)}}{M_{P l}^{3}} S_{8} S_{7} \varphi_{21}\left\langle\varphi_{27} \varphi_{30} \varphi_{4}\right\rangle+\frac{\alpha_{6}^{(3)}}{M_{P l}^{3}} S_{4} S_{6} \varphi_{20}\left\langle\varphi_{27} \varphi_{30} \varphi_{12}\right\rangle+\frac{\alpha_{6}^{(4)}}{M_{P l}^{3}} S_{8} S_{6} \varphi_{20}\left\langle\varphi_{27} \varphi_{30} \varphi_{4}\right\rangle
\end{aligned}
$$

\section{A. General implications}

This model has many features in common with the previous case: (i) there are many massless ordinary fermions and exotics for which we have no apparent mechanism to give masses; (ii) the string scale Yukawa couplings display (unrealistic) $t-b$ and $\tau-\mu$ unification [at least in the scenario where $U(1)^{\prime}$ is broken at the electroweak scale] with the respective Yukawa couplings $g$ and $g / \sqrt{2}$; (iii) there is no effective "canonical" $\mu$ term in the superpotential (however, there is a possibility of a "non-canonical" $\mu$ term); (iv) the $U(1)^{\prime}$ charges are not family universal. On the other hand, there are additional features unique to this flat direction.

\section{Implications of $L$ and $B$ violating couplings}

Proton decay. In this model, there are two effective couplings of the type $u d^{c} d^{c}$, arising from fourth order terms in the original superpotential [the seventh and eighth terms in Eq. (25)]. The superpotential also has two lepton number violating couplings of the type $e^{c} h h$ [the 11th and 12th terms 


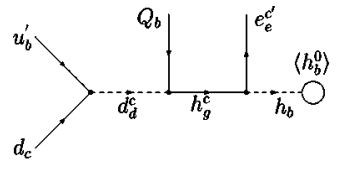

(a)

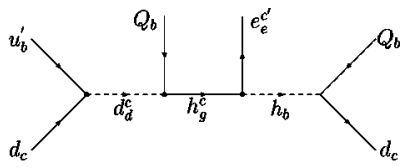

(b)
FIG. 3. Proton decay diagrams for (a) $\left\langle h_{b}^{0}\right\rangle \neq 0$, (b) $\left\langle h_{b}^{0}\right\rangle$ $=0 . \quad u_{b}^{\prime}, d_{c}$ and $d_{b}$ are the congugate of $u_{b}^{c^{\prime}}, d_{c}^{c}$ and $d_{b}^{c}$.

in Eq. (25)], since we identify both $h_{b}$ and $h_{g}$ as Higgs doublets due to their couplings to ordinary quarks. ${ }^{9}$ As a result of these two types of couplings, there is potential danger for proton decay, illustrated by the diagrams displayed in Fig. 3. ${ }^{10}$ The decay rate depends on the masses of the fields involved, and on whether $h_{b}^{0}$ has a VEV, and thus it depends on the details of the soft breaking as well as on a particular identification of the particles participating in the process; i.e., some participants can be identified with the exotics and/or the second and third family fermions. In particular, $e_{e}^{c^{\prime}}$ could be exotics, which would suppress the decay. However, they are massless within our approach; i.e., the model is not sufficiently realistic to make these exotic states significantly massive.

The proton decay rate will be much too fast unless some of the external legs almost completely decouple from the first two families. Defining $U_{a}^{(4)}$ as the product of the unitary matrix elements relating the four external legs in Fig. 3(a) to the states relevant to proton decay, and $U_{b}^{(6)}$ as the corresponding product of six matrix elements for Fig. 3(b), one requires

$$
\begin{aligned}
\left\langle h_{b}^{0}\right\rangle U_{a}^{(4)} & <10^{-24} m_{\text {prop }}^{3}, \\
U_{b}^{(6)} & <10^{-15} m_{\text {prop }}^{5},
\end{aligned}
$$

where $\left\langle h_{b}^{0}\right\rangle$ and $m_{\text {prop }}$ (a mass scale for the internal propagators, typically set by the electroweak/SUSY scale) are in $\mathrm{TeV}$ and we have assumed a lifetime of $\tau>10^{34} \mathrm{yr}$.

$N-\bar{N}$ oscillations. Even if proton decay is somehow suppressed, the coupling of the type $u d^{c} d^{c}$ also implies that there are $N-\bar{N}$ oscillations via a diagram that involves gaugino exchange [see Fig. 4(a)]. Another process that can contribute involves exchanges of three virtual squarks [Fig. 4(b)], but is more model dependent since it depends on the trilinear soft supersymmetry breaking term associated with $u d^{c} d^{c}$. (For a recent review of $N-\bar{N}$ oscillations see [40].) Since all the effective trilinear terms in the superpotential are

${ }^{9}$ We could instead interpret $h_{b}$ as a lepton doublet. In that case the $e_{e}^{c^{\prime}} h_{g} h_{b}$ term would conserve lepton number. However, the $L$-violation would then show up elsewhere; e.g., the fifth term in Eq. (25) would correspond to an $L$-violating leptoquark interaction.

${ }^{10}$ For some other flat directions there is, along with the baryon violating term of the type $u d^{c} d^{c}$, also a lepton violating term of the type $Q d^{c} h$ (where $h$ is a lepton doublet); e.g., the $\left.P_{1} P_{2} P_{3}\right|_{F}$ direction has such a term. In this case proton decay takes place via an effective dimension- 6 operator and is even harder to suppress.

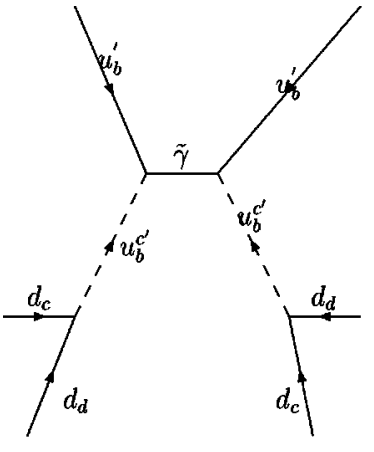

(a)

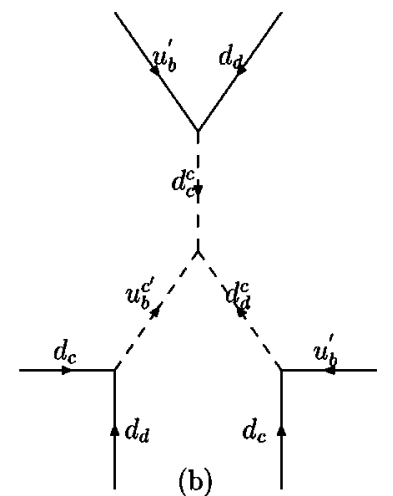

FIG. 4. Diagrams for the $\Delta B=2$ processes $N \rightarrow \bar{N}$ (neutron oscillation) or $\Delta B=2$ nuclear decay. The tri-scalar vertex in (b) corresponds to a soft supersymmetry breaking " $A$-term." Here $u_{b}^{\prime}, d_{c}$ and $d_{b}$ are the congugate of $u_{b}^{c^{\prime}}, d_{c}^{c}$ and $d_{b}^{c}$.

fixed for this flat direction, and this coupling appears at the fourth order and is not suppressed, the only suppression is due to the identification of the fields in the coupling with exotics and/or second and third family fermions. (For some other flat directions, e.g., $\left.P_{1}^{\prime} P_{2} P_{3}\right|_{F}$, the trilinear coupling depends on one free VEV, allowing for additional suppressions if that VEV is small.)

From the experimental limits on oscillations and $\Delta B=2$ nuclear decays [40], one finds

$$
U_{c}^{(6)} \lesssim 10^{-11} m_{\text {prop }}^{5},
$$

where $U_{c}^{(6)}$ is the product of the unitary matrix elements for the six external fermions onto the neutron states and $m_{\text {prop }}$ is in $\mathrm{TeV}$. Again one would need one or more of the states to almost decouple from the first family.

$R$-parity violating processes. The lepton- and baryonviolating couplings imply that there is no stable LSP. E.g., if one assumes that the LSP is a gaugino, the baryon-violating coupling allows for a decay of a gaugino into three quarks via the exchange of a virtual squark, as shown in Fig. 1(b), and the lepton-violating coupling into three leptons via a virtual slepton, by a diagram analogous to Fig. 1(a).

\section{Textures}

The Yukawa couplings for the ordinary quarks display a possible texture for the down-type quarks, if the Higgs doublets $h_{c}, h_{b}$, and $h_{g}$ all acquire VEV's. In this case, the mass matrix for the down-type quarks is ${ }^{11}$

$$
M=\left(\begin{array}{ccc}
\left\langle h_{g}\right\rangle \frac{\alpha_{5}^{(3)} x^{2}}{\sqrt{2} M_{P l}^{2}} & \left\langle h_{b}\right\rangle \frac{\alpha_{5}^{(6)} x^{2}}{\sqrt{2} M_{P l}^{2}} & 0 \\
\left\langle h_{g}\right\rangle \frac{\alpha_{5}^{(4)} x^{2}}{\sqrt{2} M_{P l}^{2}} & \left\langle h_{b}\right\rangle \frac{\alpha_{5}^{(5)} x^{2}}{\sqrt{2} M_{P l}^{2}} & 0 \\
0 & 0 & g\left\langle h_{c}\right\rangle
\end{array}\right),
$$

\footnotetext{
${ }^{11}$ It is possible for $\left\langle h_{g}\right\rangle$ and $\left\langle h_{b}\right\rangle$ to accquire phases by a spontaneous breaking of $C P$ for some symmetry breaking patterns [27]. However, these phases can be absorbed into the definition of the mass eigenstate quarks, so that $U_{C K M}$ is real for this model.
} 
and the mass terms in the Lagrangian are

$$
-L_{\text {mass }}=\left(Q_{a}, Q_{b}, Q_{c}\right) M\left(\begin{array}{c}
d_{a / c}^{c} \\
d_{d}^{c} \\
d_{b}^{c}
\end{array}\right) .
$$

The state $d_{a}^{c}$ is the partner of the exotic $\mathcal{D}_{a}$; both remain massless in this model. It may be possible for all four Higgs doublets $\bar{h}_{c}, h_{c}, h_{b}$ and $h_{g}$ to acquire non-zero VEV's. Then the mass matrix for the down-type quarks has the most general form given in Eq. (30), which by inspection demonstrates that there is no mixing between the third family and the first two families. In general, there are three massive states: one heavy (bottom) quark, and two lighter quarks due to suppressions from the higher order terms. One of them may be massless if the string coefficients $\alpha_{5}^{(3)}=\alpha_{5}^{(4)}$ and $\alpha_{5}^{(5)}=\alpha_{5}^{(6)}$, which also results in nearly maximal mixing in the case in which $\left\langle h_{b}\right\rangle \sim\left\langle h_{g}\right)$. If either $h_{b}$ or $h_{g}$ does not acquire a VEV, there will be one massless and two massive eigenstates (once again, with a hierarchy of masses). Clearly, if both $h_{b}$ and $H_{g}$ do not acquire VEV's, the down quark states of the first two families will remain massless.

It is convenient to define the dimensionless ratios (for the case $\left\langle h_{c}\right\rangle \neq 0$ )

$$
\begin{gathered}
\kappa_{3,4}=\frac{\left\langle h_{g}\right\rangle}{\left\langle h_{c}\right\rangle} \frac{\alpha_{5}^{(3,4)} x^{2}}{\sqrt{2} g M_{P l}^{2}} \sim 0.1 \frac{\left\langle h_{g}\right\rangle}{\left\langle h_{c}\right\rangle}, \\
\kappa_{5,6}=\frac{\left\langle h_{b}\right\rangle}{\left\langle h_{c}\right\rangle} \frac{\alpha_{5}^{(5,6)} x^{2}}{\sqrt{2} g M_{P l}^{2}} \sim 0.1 \frac{\left\langle h_{b}\right\rangle}{\left\langle h_{c}\right\rangle},
\end{gathered}
$$

where the numerical values use the estimates of $\alpha_{5}$ from Sec. II. Then, neglecting the running of the Yukawas down to low energies, the quark masses are

$$
\begin{aligned}
& \left(m_{u}, m_{c}, m_{t}\right)=(0,0,1) \times g\left\langle\bar{h}_{c}\right\rangle, \\
& \left(m_{d}, m_{s}, m_{b}\right)=\left(\delta_{1}, \delta_{2}, 1\right) \times g\left\langle h_{c}\right\rangle,
\end{aligned}
$$

in which $\delta_{1,2}$ are the mass eigenvalues

$$
\begin{aligned}
\delta_{1,2}= & {\left[\frac { \sum _ { i = 3 } ^ { 6 } \kappa _ { i } ^ { 2 } } { 2 } \mp \frac { 1 } { 2 } \left(\left(\kappa_{6}^{2}+\kappa_{3}^{2}-\kappa_{4}^{2}-\kappa_{5}^{2}\right)^{2}\right.\right.} \\
& \left.\left.+4\left(\kappa_{6} \kappa_{5}+\kappa_{3} \kappa_{4}\right)^{2}\right)^{1 / 2}\right]^{1 / 2} .
\end{aligned}
$$

Although this is not fully realistic, it illustrates the possibility of a realistic $m_{s} / m_{b}$ ratio due to the contribution of the fifth order terms. For $\kappa_{3}=\kappa_{4}$ and $\kappa_{5}=\kappa_{6}$, one has $m_{d}=0$, as expected. However, a small $m_{d} / m_{s}$ can emerge if $\kappa_{3} \neq \kappa_{4}$ or $\kappa_{5} \neq \kappa_{6}$. One does not expect the $\alpha$ 's of the same order to be equal in general, leading to the possibility of a small $m_{d} / m_{s}$ even though they both are from dimension 5 operators. The expected ratios of the doublet VEV's will be discussed in [27]. It is straightforward to determine the Cabibbo-
Kbayashi-Maskawa (CKM) quark mixing matrix corresponding to this texture. One finds

$$
U_{\mathrm{CKM}}=\left(\begin{array}{ccc}
\cos \theta_{c} & \sin \theta_{c} & 0 \\
-\sin \theta_{c} & \cos \theta_{c} & 0 \\
0 & 0 & 1
\end{array}\right),
$$

in which the mixing angle $\theta_{c}$ if given by

$$
\tan \left(2 \theta_{c}\right)=\frac{2\left(\kappa_{3} \kappa_{4}+\kappa_{5} \kappa_{6}\right)}{\kappa_{3}^{2}+\kappa_{6}^{2}-\kappa_{4}^{2}-\kappa_{5}^{2}} .
$$

For $\kappa_{3}=\kappa_{4}$ and $\kappa_{5}=\kappa_{6}$, one obtains maximal mixing and $\theta_{c}=\pi / 4$. Relaxing the equality of the $\alpha_{5}$ 's one obtains a more realistic value for $\theta_{c}$, as well as $m_{d} / m_{s} \neq 0$. However, the relation $\theta_{c} \sim\left(m_{d} / m_{s}\right)^{1 / 2}(\sim 0.2)$ does not hold except for special values of the parameters.

As for the texture in the lepton sector, if $h_{g}$ acquires a non-zero VEV, then the terms $e_{b}^{c} h_{g} h_{a}$ and $e_{i}^{c} h_{g} h_{a}$ in Eq. (25) can (slightly) break the (unrealistic) $\tau$ - $\mu$ degeneracy. However, the electron remains massless in this scenario.

\section{Running of the gauge couplings}

The mass spectrum for the quark sector for this model is the same as in the previous case; hence, we adopt the same strategy for the running of the gauge couplings. The $\beta$ functions, as listed in Table $\mathrm{V}$, are similar to those of the $P_{1}^{\prime} P_{2}^{\prime} P_{3}^{\prime}$ direction. Again we choose $\alpha_{s}=0.12$ at $M_{Z}$ and run the couplings to the string scale to determine the same value of $g$ $=0.80$. (The number of massless colored fields is the same as in the previous example.) We find the values $g_{1}=0.40$ (which includes $k_{Y}=11 / 3$ factor) and $g_{2}=0.46\left(k_{2}=1\right)$ at the electroweak scale, yielding $\sin ^{2} \theta_{W}=0.17$.

\section{B. Possible $U(1)^{\prime}$ symmetry breaking scenarios}

In this model, $\varphi_{25}$ remains massless at the string scale [see Table IV(b)]. This field, which has $U(1)^{\prime}$ charge opposite in sign to all the other singlet fields [with nonzero $U(1)^{\prime}$ charge], is required for ensuring the $U(1)^{\prime} D$-flat direction, and thus is necessary for the intermediate scale symmetry breaking scenario, as discussed in [33]. The $U(1)^{\prime} D$-flat direction involves $\varphi_{25}$ and one of the singlet fields $\left\{\varphi_{18}, \varphi_{19}, \varphi_{20}, \varphi_{21}, \varphi_{22}\right\}$. The couplings of these singlet fields seem to indicate that an intermediate scale flat direction involving $\varphi_{20}$ or $\varphi_{21}$ is potentially dangerous, as it would decouple $\bar{h}_{c}$ and, hence, the top quark coupling. However, the $D$-flat directions involving $\varphi_{20}, \varphi_{21}$ are not $F$-flat at the renormalizable level. In particular, they are lifted by the couplings $\varphi_{25} \varphi_{20} \varphi_{28}$ and $\varphi_{25} \varphi_{21} \varphi_{29}$, respectively, and thus the symmetry breaking scale would take place at the electroweak scale.

If one can ensure that the supersymmetry breaking mass squares of $\varphi_{20}$ and $\varphi_{21}$ stay positive at low energies, while $m_{\varphi_{25}}^{2}+m_{\varphi_{18 / 19 / 22}}^{2}$ is negative, then we can have intermediate scale symmetry breaking along the $D$-flat direction(s) $\varphi_{25}$ $+\varphi_{18 / 19 / 22}$. 
Using gauge invariance arguments and string selection rules, we find that there are no higher dimensional nonrenormalizable operators (NRO's) in the superpotential involving self-couplings of the singlets in the intermediate scale $D$-flat direction, which in principle could stabilize the flat direction and determine the intermediate scale of the symmetry breaking. Hence, in this model the symmetry breaking is purely radiative, with the scale $\mu_{R A D}$ very close to the scale at which the sum of the mass squares $m_{\varphi_{25}}^{2}$ $+m_{\varphi_{18 / 19 / 22}}^{2}$ crosses zero [33].

After the intermediate scale symmetry breaking, the electroweak symmetry breaking has a few novel features, different from the previous case:

(i) In this case, $h_{c}$ is heavy and decouples, and so the bottom quark Yukawa is absent in the theory. The first and second families can have electroweak scale masses if $h_{b}$ and $h_{g}$ acquire VEV's. In addition, the decoupling of $h_{c}$ makes $\mu$ and $\tau$ massless at the electroweak scale, since couplings $h_{a} h_{c} e_{a}^{c}$ and $h_{d} h_{c} e_{f}^{c}$ all disappear. In addition, $\varphi_{20}, \varphi_{21}, \varphi_{28}$ and $\varphi_{29}$ all acquire intermediate scale masses. As a result, all the trilinear "half" $\mu$-terms in Eq. (25) vanish.

(ii) The running of the gauge couplings has to be modified in order to take into account the decoupling of the heavy states. However, the complete determination of the fields with intermediate scale masses requires a detailed knowledge of the relevant NRO's, and is beyond the scope of the paper.

(iii) In principle, it is also possible to give the total singlets (with $Q_{Y}=Q^{\prime}=0$ ) intermediate scale VEV's; since they do not have any $D$ - term, the symmetry breaking scenario is similar to the case with the charged SM singlets in a $D$-flat direction. In this model, the only total singlets which have Yukawa couplings (and hence their mass squares can be driven negative radiatively) are $\varphi_{28}$ and $\varphi_{29}$. However, these fields couple to $\varphi_{25}$ at the effective trilinear level, which give rise to effective $F$-terms that push the VEV's down to the electroweak scale. Therefore, $\varphi_{28}$ and $\varphi_{29}$ cannot be involved in the intermediate scale flat direction at the same time as $\varphi_{25}$.

These possibilities are discussed in detail for specific Ansätze for the soft supersymmetry breaking mass terms in [27], while the implications for generating the effective $\mu$ term as well as ordinary and exotic fermion masses via higher dimensional operators [33] are deferred to a future study.

\section{CONCLUSIONS}

In this paper we have given a thorough investigation of the physics implications for the observable sector of the CHL5 model from the "top-down" approach. For this model a complete classification of the $D$ - and $F$-flat directions due to non-zero VEV's of the non-Abelian singlets was found [18]; these directions were shown to be flat to all orders in string perturbation. Along all these flat directions there is at least one additional $U(1)^{\prime}$ factor (along with the SM gauge group). We chose two particular flat directions as representative examples which exhibit the type of physics phenom- ena characteristic for the quasi-realistic string vacua based on free-fermionic constructions.

For each of the representative directions we found a complete massless spectrum and the trilinear terms in the observable sector of the superpotential. (We also determined the terms of the observable sector fields to the hidden ones that may play a role in the RGE analysis.) Gauge invariant bilinear and trilinear terms were first determined to all orders in fields with non-zero VEV's along a particular flat direction. The subsequent string calculation for a particular gauge invariant term then determines whether this term is indeed there. Notably, the world-sheet constraints of string theory disallow a large number of gauge invariant terms; thus, there are in general fewer terms than expected. These "string selection rules" have in general important physics implications:

Massless spectrum. Along with the MSSM particle content there are a large number of additional massless (at the string scale) exotics. In the case of electroweak scale $U(1)^{\prime}$ breaking, the exotic fermions are light compared with the electroweak scale, which is clearly excluded by experiment. This feature seems to pose a serious problem in the search for a realistic string vacuum.

Surprisingly, the type of massless particle content that survives for the two representative flat directions, combined with the higher Kač-Moody level for $U(1)_{Y}$, still allows for a gauge coupling unification with a prediction for $\sin \theta_{W}$ $\sim 0.16$ that, while not consistent with experiment, is not too far away from the experimental value.

Trilinear couplings. The string models based on a free fermionic construction possess the feature that nonzero trilinear terms at the third order are of the order of the gauge coupling, and thus large. For the particular model discussed they are equal to $g$ or $g / \sqrt{2}$. [Such large trilinear terms facilitate the radiative symmetry breaking scenarios of the SM and $U(1)^{\prime}$ gauge structure.] Importantly, the trilinear terms surviving at the fourth order turn out to have effective couplings that are comparable to those at the third order [37]. Only at the fifth and higher orders does the suppression of these coupling become significant, i.e., of order $1 / 10$ and smaller.

Again, the number of allowed trilinear terms in the superpotential is significantly smaller than allowed by gauge invariance, which has a number of implications:

Fermion textures. The CHL5 model possesses a distinct feature that in the quark sector only one up-type and one down-type quark have Yukawa couplings at the third order, while in the lepton sector generically two $e$-type couplings have Yukawa couplings at the third order, so that at the string scale there is $t-b$ and $\tau$ - $\mu$ universality with the respective couplings $g$ and $g / \sqrt{2}$. While $t-b$ universality is consistent with experiment for sufficiently large $\tan \beta$, the ratio of $b$ and $\tau$ Yukawa couplings is somewhat large, and $\tau$ - $\mu$ universality is clearly not physical. For certain flat directions (e.g., the first example) there is no further texture in the fermion mass matrix. On the other hand, in other directions (e.g., the second example) the texture is induced at a higher orderhowever, only in the down quark sector, and only certain entries arising at a specific order (e.g., for the second ex- 
ample, at the fifth order, but with the $d$-quark remaining massless). These features are modified in the intermediate scale case, in which the $h_{c}$ acquires an intermediate scale mass (and no VEV), and most of the fermion masses would have to be generated by higher dimensional operators.

$\mu$ parameter. In principle, there is the possibility of having a standard effective $\mu$-term at the electroweak scale, due to the trilinear couplings of the two standard Higgs doublets (which respectively couple to the $t$ - and $b$-quarks) to the SM singlet(s) which acquire non-zero VEV's at the electroweak scale [and, if they are charged under the additional $U(1)^{\prime}$, give mass to the $Z^{\prime}$ ]. It turns out that there is no such canonical $\mu$-term in these examples. However, there are "noncanonical" $\mu$-terms that involve a standard Higgs doublet with a coupling to the $t$-quark and another Higgs field (which may couple to the $s$ - or $d$-quark at high orders). This noncanonical $\mu$-parameter has interesting implications for the neutralino and chargino sector mass patterns.

Baryon- and lepton-number violating couplings. In general such couplings are present; they may induce proton decay, $N-\bar{N}$ oscillations and/or leptoquark couplings and break $R$-parity, so that there is no stable LSP. For specific directions such couplings are absent or could be suppressed within a specific SM symmetry breaking scenario.

The $U(1)^{\prime}$ symmetry breaking pattern can be either at an intermediate or at the electroweak scale. It depends on the $U(1)^{\prime}$ charges and the type of trilinear couplings of the massless SM singlets. E.g., for the first representative direction all the $U(1)^{\prime}$ charges of the massless SM singlets have the same sign; thus, there is no $D$-flat $U(1)^{\prime}$ direction, and the breaking necessarily takes place at the electroweak scale. The particular values of the $U(1)^{\prime}$ charges for the light particle spectrum (which are family non-universal), imply new experimental constraints on this $Z^{\prime}$. On the other hand, in the second example there is an additional massless SM singlet with the opposite sign of $U(1)^{\prime}$ charge from the other singlets, and $F$-flat $U(1)^{\prime}$-directions for the trilinear couplings, and thus symmetry breaking can take place at an intermediate scale. It turns out to be purely radiative in origin, because of the absence of the relevant nonrenormalizable terms in the superpotential.

Further specific properties of the symmetry breaking patterns, and the corresponding mass spectrum at the electroweak scale, depend on the soft supersymmetry breaking mass terms introduced by hand at the string scale. Specific examples with a realistic electroweak SM and $U(1)^{\prime}$ breaking pattern will be investigated in [27].

While the trilinear couplings in the hidden sector were not explored to all orders in the VEV's of the fields in a particular flat direction, the terms at the third and fourth order are known [11]. (Terms at higher orders are suppressed.) Further investigation of the implications of the hidden sector is underway.

The work presented in this paper opens a number of further avenues for investigation. Specifically, the study of non- renormalizable terms in the superpotential is needed. In this case the investigation is complicated by the fact that along with the direct determination of the non-renormalizable terms in string theory there are additional induced terms due to the decoupling effects of the fields that became heavy after vacuum restabilization. (For a detailed investigation of these decoupling effects see [25].) Further study of the corrections to the Köhler potential after vacuum restabilization is also needed. These effects may have further implications for the mass spectrum in the intermediate scale $U(1)^{\prime}$ breaking scenarios. In particular, a number of exotic fields could acquire mass at a scale larger than the electroweak one. In addition, additional entries in the fermion textures can appear and effective $\mu$ term can be generated [33].

The techniques for systematic classification of $D$ - and $F$-flat directions $[17,18]$ for perturbative heterotic string vacua with anomalous $U(1)^{\prime}$ and the subsequent determination of the mass spectrum and coupling of the restabilized vacua, as investigated in this paper, are general, and can be applied immediately to the study of other quasi-realistic models, which is also underway. These techniques may also be applied to the study of non-perturbative heterotic string vacua with anomalous $U(1)^{\prime}[41]$.

The models discussed in this paper are not fully realistic, and contain such features as very light or massless exotic fermions, charginos, and neutralinos, an unwanted $\tau-\mu$ universality and undesirable ratio of the $b$ - and $\tau$-string scale Yukawa couplings, unrealistic fermion textures, and possible proton decay, etc. However, they also contain at least the gauge structure and particle content of the MSSM, and illustrate a number of features of this class of string models that are likely to be present in many other string models, including nonperturbative ones. These include additional $Z^{\prime}$ bosons, which may have family-nonuniversal couplings and which may have masses either at the electroweak scale or an intermediate scale, exotic fermions and their scalar partners, approximate gauge unification, the possibility of effective non-standard $\mu$ terms, an extended neutralino or chargino spectrum, the possibility of baryon and/or lepton number violation, and $R$-parity violation, leading to the absence of a stable LSP, leptoquark couplings, and non-standard chargino or neutralino decays.

\section{ACKNOWLEDGMENTS}

We would especially like to thank J. Lykken for making available to us the program that generates the massless spectrum of free fermionic string vacua as well as the original program that calculates the superpotential couplings. We would also like to thank him for numerous discussions and guidance that enabled us to determine fully the relevant superpotential couplings. M.C., L.E. and J. W. would also like to thank Fermilab, where part of the work was done, for hospitality. L.E. also thanks Gordon Kane for helpful discussions. This work was supported in part by U.S. Department of Energy Grant No. DOE-EY-76-02-3071. 
[1] P. Candelas, G.T. Horowitz, A. Strominger, and E. Witten, Nucl. Phys. B258, 46 (1985).

[2] W. Lerche, D. Lust, and A.N. Schellekens, Phys. Lett. B 181, 71 (1986); Nucl. Phys. B287, 477 (1987).

[3] I. Antoniadis, C. Bachas, C. Kounnas, and P. Windey, Phys. Lett. B 171, 51 (1986); I. Antoniadis, C. Bachas, and C. Kounnas, Nucl. Phys. B289, 87 (1987); I. Antoniadis and C. Bachas, ibid. B298, 586 (1988).

[4] H. Kawai, D. Lewellen, and S.-H. Tye, Nucl. Phys. B288, 1 (1987); H. Kawai, D. Lewellen, J.A. Schwartz, and S.-H. Tye, ibid. B299, 431 (1988).

[5] L. Dixon, J.A. Harvey, C. Vafa, and E. Witten, Nucl. Phys. B261, 678 (1985); B274, 285 (1986); L. Ibáñez, H.P. Nilles, and F. Quevedo, Phys. Lett. B 187, 25 (1987); Nucl. Phys. B192, 332 (1987).

[6] L. Ibáñez, J.E. Kim, H.P. Nilles, and F. Quevedo, Phys. Lett. B 191, 282 (1987); J.A. Casas and C. Muñoz, ibid. 209, 214 (1988); 214, 157 (1988); J.A. Casas, E. Katehou, and C. Muñoz, Nucl. Phys. B317, 171 (1989); A. Font, L. Ibáñez, H.P. Nilles, and F. Quevedo, Phys. Lett. B 210, 101 (1988); A. Chamseddine and M. Quirós, ibid. 212, 343 (1988); Nucl. Phys. B316, 101 (1989); A. Font, L. Ibáñez, F. Quevedo, and A. Sierra, ibid. B331, 421 (1990).

[7] B. Greene, K. Kirlin, P. Miron, and G.G. Ross, Nucl. Phys. B278, 667 (1986); B292, 606 (1987).

[8] I. Antoniadis, J. Ellis, J. Hagelin, and D.V. Nanopoulos, Phys. Lett. B 231, 65 (1989); I. Antoniadis, G.K. Leontaris, and J. Rizos, ibid. 245, 161 (1990); A.E. Faraggi, Nucl. Phys. B387, 239 (1992); hep-th/9708112; A.E. Faraggi and D.V. Nanopoulos, Phys. Rev. D 48, 3288 (1993).

[9] A. Faraggi, D.V. Nanopoulos, and K. Yuan, Nucl. Phys. B335, 347 (1990); A. Faraggi, Phys. Rev. D 46, 3204 (1992).

[10] A. Faraggi, Phys. Lett. B 278, 131 (1992); Nucl. Phys. B403, 101 (1993); Phys. Lett. B 339, 223 (1994).

[11] S. Chaudhuri, G. Hockney, and J. Lykken, Nucl. Phys. B469, 357 (1996); erratum (in preparation).

[12] V. Kaplunovsky, Nucl. Phys. B307, 145 (1988); B382, 436(E) (19992).

[13] K.R. Dienes, Phys. Rep. 287, 447 (1997).

[14] L. Dixon, E. Martinec, D. Friedan, and S. Shenker, Nucl. Phys. B282, 13 (1987).

[15] L. Dixon and M. Cvetič (unpublished); M. Cvetič, in Proceedings of Superstrings, Cosmology, and Composite Structures, College Park, Maryland, 1987, edited by S.J. Gates and R. Mohapatra (World Scientific, Singapore, 1987); Phys. Rev. Lett. 59, 2829 (1987).

[16] D. Bailin, D. Dunbar, and A. Love, Phys. Lett. B 219, 76 (1989); S. Kalara, J. López, and D.V. Nanopoulos, ibid. 245, 421 (1990); Nucl. Phys. B353, 650 (1991); A. Faraggi, ibid. B487, 55 (1997).

[17] G. Cleaver, M. Cvetič, J.R. Espinosa, L. Everett, and P. Langacker, Nucl. Phys. B525, 3 (1998).

[18] G. Cleaver, M. Cvetič, J.R. Espinosa, L. Everett, and P. Langacker, hep-th/9805133.

[19] M. Dine, N. Seiberg, and E. Witten, Nucl. Phys. B289, 585 (1987).

[20] J. Atick, L. Dixon, and A. Sen, Nucl. Phys. B292, 109 (1987);
M. Dine, I. Ichinose, and N. Seiberg, ibid. B293, 253 (1987);

M. Dine and C. Lee, ibid. B336, 317 (1990).

[21] J. Atick and A. Sen, Nucl. Phys. B296, 157 (1988).

[22] L. Dixon and V. Kaplunovsky (unpublished).

[23] J.A. Casas and C. Muñoz, Phys. Lett. B 216, 37 (1989); M. Yamaguchi, H. Yamamoto, and T. Onogi, Nucl. Phys. B327, 704 (1989); J.A. Casas, J.M. Moreno, C. Muñoz, and M. Quirós, ibid. B328, 272 (19891); J. López and D.V. Nanopoulos, Phys. Lett. B 245, 111 (1990); C.J.H. Lee, Ph.D. thesis, 1992, Report No. UMI-92-24830; A. Faraggi, Nucl. Phys. B387, 239 (1992); Phys. Lett. B 302, 202 (1993); Nucl. Phys. B407, 57 (1993); H. Nakano, Report No. KUNS-1257, hep-th/9404033; T. Kobayashi and H. Nakano, Nucl. Phys. B496, 103 (1997); G. Cleaver and A.E. Faraggi, Report No. UFIFT-HEP-97-28, UPR-0773-T, hep-ph/9711339.

[24] Y. Kawamura and T. Kobayashi, Phys. Lett. B 375, 141 (1996); A. Faraggi and E. Halyo, Int. J. Mod. Phys. A 11, 2357 (1996); G. Dvali and A. Pomarol, Phys. Rev. Lett. 77, 3728 (1996); Nucl. Phys. B522, 3 (1998); P. Binétruy and E. Dudas, Phys. Lett. B 389, 503 (1996); R.N. Mohapatra and A. Riotto, Phys. Rev. D 55, 1138 (1997); 55, 4262 (1997); Z. Berezhiani and Z. Tavartkiladze, Phys. Lett. B 396, 150 (1997); K. Choi, E. Chun, and H. Kim, ibid. 394, 89 (1997); P. Binétruy, N. Irges, S. Lavignac, and P. Ramond, ibid. 403, 38 (1997); R.J. Zhang, ibid. 402, 101 (1997); A. Nelson and D. Wright, Phys. Rev. D 56, 1598 (1997); M.M. Robinson and J. Ziabicki, Report No. EFI-97-22, hep-ph/9705418; N.K. Sharma, P. Saxena, S. Smith, A.K. Nagawat, and R.S. Sahu, Phys. Rev. D 56, 4152 (1997); N. Irges and S. Lavignac, Phys. Lett. B 424, 293 (1998); A. Faraggi and J. Pati, Nucl. Phys. B526, 21 (1998); A. Faraggi, Phys. Lett. B 426, 315 (1998); N. Irges, S. Lavignac, and P. Ramond, Phys. Rev. D 58, 035003 (1998); A. Pomarol, Report No. PRINT-98-021, 1997.

[25] M. Cvetič, L. Everett, and J. Wang, hep-ph/9807321.

[26] E. Witten, Nucl. Phys. B268, 79 (1986); M. Dine and N. Seiberg, Phys. Rev. Lett. 57, 2625 (1986).

[27] G. Cleaver, M. Cvetič, J.-R. Espinosa, L. Everett, J. Wang, and P. Langacker, Report No. UPR-814-T (in preparation).

[28] M. Cvetič and P. Langacker, Phys. Rev. D 54, 3570 (1996); Mod. Phys. Lett. A 11, 1247 (1996); in Perspectives on Supersymmetry, edited by G. L. Kane (World Scientific, Singapore, 1998), p. 312.

[29] D. Suematsu and Y. Yamagishi, Int. J. Mod. Phys. A 10, 4521 (1995).

[30] M. Cvetič, D.A. Demir, J.R. Espinosa, L. Everett, and P. Langacker, Phys. Rev. D 56, 2861 (1997); Cleaver et al. [33].

[31] P. Langacker and J. Wang, Phys. Rev. D 58, 115010 (1998).

[32] E. Keith and E. Ma, Phys. Rev. D 56, 7155 (1997).

[33] G. Cleaver, M. Cvetič, J.R. Espinosa, L. Everett, and P. Langacker, Phys. Rev. D 57, 2701 (1998).

[34] See, e.g., M.A. Luty and W. Taylor IV, Phys. Rev. D 53, 3399 (1996), and references therein.

[35] T. Gherghetta, C. Kolda, and S. Martin, Nucl. Phys. B468, 37 (1996).

[36] P. Binétruy, N. Irges, S. Lavignac, and P. Ramond, Phys. Lett. B 403, 38 (1997).

[37] M. Cvetič, L. Everett, and J. Wang, hep-ph/9808321. 
[38] L.J. Hall and M. Suzuki, Nucl. Phys. B231, 419 (1984): T. Banks et al., Phys. Rev. D 52, 5319 (1995); R. Hempfling, Nucl. Phys. B478, 3 (1996); B. de Carlos and P.L. White, Phys. Rev. D 54, 3427 (1996); A. Yu Smirnov and F. Vissani, Nucl. Phys. B460, 37 (1996); R.M. Borzumati et al., Phys. Lett. B 384, 123 (1996); H.P. Nilles and N. Polonsky, Nucl. Phys. B499, 33 (1997); E. Nardi, Phys. Rev. D 55, 5772 (1997).
[39] P. Langacker and N. Polonsky, Phys. Rev. D 49, 1454 (1994); M. Carena et al., Nucl. Phys. B426, 269 (1994); V. Barger, M.S. Berger, and P. Ohmann, Phys. Rev. D 47, 1093 (1993).

[40] R. Mohapatra, Proceedings of Future Prospects of Baryon Instability Search, Oak Ridge, 1996, p. 73.

[41] G. Aldazabal, A. Font, L.E. Ibáñez, and G. Violero, Nucl. Phys. B536, 29 (1998); Z. Kakushadze, Phys. Lett. B 434, 269 (1998); J. Lykken, E. Poppitz, and S.P. Trivedi, hep-th/9806080. 